\title{
A Multi-kilogram Capacity Calorimeter For Heterogeneous Materials
}

K. L. Churney, A. E. Ledford, M. L. Reilly, and E. S. Domalski

National Bureau of Standards Gaithersburg, MD 20899

\begin{abstract}
A large capacity calorimeter was designed and constructed in order to determine the enthalpies of combustion of kilogram-size samples of municipal solid waste (MSW) in flowing oxygen near atmospheric pressure. The combustion of the organic fraction of the samples was complete to greater than $99.9+\%$. The percent coefficient of variation ( 100 $x$ standard deviation/average), $\% C V$, of calibration measurements using microcrystalline cellulose was $0.2 \%$. The $\% \mathrm{CV}$ of the measurements of the en-
\end{abstract}

thalpy of combustion of a processed MSW sample was $0.4 \%$. The combined systematic errors due to departure from usual design standards and conventional operating procedures is estimated to be less than $0.4 \%$ of the calorific value.

Key words: calorific value; combustion; flow calorimeter; kilogram capacity calorimeter; municipal solid waste.

Accepted: April 24, 1986

\section{Introduction}

Proof that small test samples are representative of a large parent material is essential to the credibilitity of test methods used to characterize the material. The importance and cost of this proof increases as the heterogeneity of the parent material becomes greater. The calorimeter described in this paper was built to determine if the calorific values of kilogram-size parent and gram-size test samples are the same within $1 \%$ for an extremely heterogeneous material-municipal solid waste (MSW).

A description of the industrial problem concerning the calorific value of MSW and the response to solve this problem by the National Bureau of Stan- dards (NBS), U.S. Department of Energy (DoE), and the U.S. Environmental Protection Agency (EPA) are summarized in section 2. The calorimeter and its auxiliary measurement systems for determining the calorific value of MSW are described in section 3. The main features of a typical experiment, the methods of data reduction, and typical results are given in section 4 . With this background, the design basis and the performance characteristics of the calorimeter are discussed in section 5 .

The combustion flow calorimeter designed for the measurements is novel in three respects: 1) It can accommodate a large solid sample. Thus, it

About the Authors: K. L. Churney, A. E. Ledford, and E. S. Domalski are with the Chemical Thermodynamics Division in the Center for Chemical Physics at NBS while M. L. Reilly is with the Temperature and Pressure Division in the Bureau's Center for Basic Standards. The work described was funded by the U.S. Department of Energy (Biofuels and Municipal Waste Technology Division) from 1977 to the present, U.S. Environmental Protection Agency (Solid and Hazardous Waste Research Division) from 1977 to 1979, and the NBS (Office of Recycled Materials) from 1977 to 1982. 
provides a unique capability to determine the enthalpy of combustion of heterogeneous solid materials for which further sampling and processing to obtain representative gram-size test samples may be costly or difficult. 2) The combustion of the solid sample is carried out in flowing oxygen near atmospheric pressure. Thus, the successful operation of this calorimeter revives a calorimetric technique for the combustion of solids that was discarded before 1900 . The technique can now be applied to other problems which require a completeness of combustion up to $99.9 \%$. 3) The design and operation depart from certain standard design criteria and conventional operating procedures employed in smaller, more accurate, stirred-water calorimeters.

\section{The Industrial Problem and the NBS-DoE Response}

Incinerator-boiler systems used to dispose of MSW are bought and sold on the basis of thermal specifications. To determine whether a system meets its thermal specifications, the calorific value of the input waste stream must be known.

At present, as well as in the past, the calorific value of MSW is determined at commercial test laboratories by bomb calorimetry using gram-size test samples. The gram-size test samples are usually prepared from kilogram-size increments. The increments are dried, sorted to remove nonmillable components (i.e., metals), and milled to $2 \mathrm{~mm}$ particle size (i.e., 95 mass percent passes through a $2 \mathrm{~mm}$ square mesh screen). In this work, the milled material is called processed MSW. The processed MSW is then subdivided, usually by coning and quartering, to obtain gram-size test samples.

For many years, combustion engineers felt that one could not sample a multi-ton quantity of MSW and extract representative gram-size test samples for bomb calorimetric measurements. The method of preparation of test samples was also in dispute because many combustion engineers believed that the composition of the test samples differed from that of the original increments. This might occur because of changes caused by excessive localized heating during milling or by nonrepresentative subdivision of processed MSW, which segregates easily. Segregation occurs because processed MSW consists of a low density component, which resembles chopped up cotton fibers, and a high density component which resembles sand. Part of the high density material is entrained in the low density material and this entrainment is nonuniform.
To address this dispute, NBS initiated a research program in collaboration with DoE and EPA to develop test procedures for accurately determining the calorific values of MSW and fuels derived from MSW. This research was also part of the NBS response to the Resource Conservation and Recovery Act of 1976. Subtitle E of this legislation mandated NBS to provide guidelines for the development of specifications for classification of materials destined for disposal.

To resolve the dispute, two questions were addressed. The first was the sampling question: How does one obtain a representative gram-size sample from a kilogram-size quantity of processed MSW? The second was the processing question: Does milling MSW down to $2 \mathrm{~mm}$ particle size alter its calorific value significantly? The remaining problem of extraction of kilogram-size increments in sufficient number and in such a manner as to characterize a multi-ton pile of MSW was considered beyond the scope of this study. However, in order to address the two questions, increments were extracted from a multi-ton stream of MSW using a selected sampling method.

An initial study of the day-to-day variability of the calorific value of MSW was carried out at NBS in $1980[1]^{1}$ using the selected sampling method. We concluded from the results of that work that the sampling and processing questions could be answered with a calorimeter in which we could burn a kilogram-size sample with a total uncertainty of less than $1 \%$ in the enthalpy of combustion.

We chose to build a calorimeter for the combustion of kilogram-size samples in flowing oxygen near atmospheric pressure rather than scale-up the conventional oxygen combustion bomb for reasons of safety. Instrumentation of a small commercial incinerator was rejected because it was felt the time required to modify such a unit and validate the calorimetry would be longer than that needed to build a new calorimeter. A small combustion calorimeter $[2,3]$ was built to demonstrate that the oxygen flow technique could be used to obtain complete combustion of pellets of MSW. The pellet mass used in the calorimeter was $25 \mathrm{~g}$. Combustion of MSW which has been compressed into a pellet rather than left in loose form was adopted because this configuration reduces the dispersion of the sample and, thus, simplifies the collection of ash. To reduce the ambiguity in the scale-up of the 25 g capacity flow calorimeter, the burning characteristics of kilogram-size pellets of unprocessed MSW were studied in a prototype combustor [4].

\footnotetext{
' Numbers in brackets indicate literature references.
} 
Unprocessed MSW in that work, and also in the present study, was prepared by withdrawing increments from the output of the primary shredder of the Baltimore County Resource Recovery Facility at Cockeysville, MD. The increments were dried at $105^{\circ} \mathrm{C}$ for 12 hours and then sorted to remove metals. The particle size was $15 \mathrm{~cm}$.

After designing, fabricating, and assembling the multi-kilogram flow calorimeter, a study consisting of 25 experiments was carried out to answer the processing and sampling questions. After drying the increments of unprocessed MSW, we attempted to prepare identical increments by hand sorting groups of four or more increments of MSW into categories (i.e., paper, metals, wood, vegetable matter, etc.) and reconstituting the categories (except for metals) into $2.5 \mathrm{~kg}$ samples having the same mass composition as the whole. Two of the $2.5 \mathrm{~kg}$ samples from each group were milled to $2 \mathrm{~mm}$ particle size and then, to obtain gram-size test samples, each of these parent samples was subdivided using a rotary riffler rather than the usual method of coning and quartering. The results, which we believe resolve the processing and sampling questions, are discussed in detail elsewhere [5]. In brief, the results are as follows:

1) The average difference of the calorific values of gram-size test samples of dry, processed MSW minus that of their kilogram-size parent of dry, processed MSW is $-0.1 \%$ of the mean calorific value of the parent sample. The imprecision ${ }^{2}$ of the average difference is $1.1 \%$ of the average calorific value and can be accounted for by the percent coefficient of variation, ${ }^{3} \% C V$, of the bomb calorimetry measurements, $0.5 \%$, and the flow calorimetry measurements, $0.4 \%$, on these MSW samples. The subdivision process using the rotary riffler is less sensitive to the effects of segregation than the subdivision process carried out by the usual method of coning and quartering.

2) The average difference in the calorific values of MSW of kilogram-size samples of dry, unprocessed MSW minus that of dry, processed MSW is $-0.5 \%$ of the average calorific value of the processed MSW. The imprecision of the difference is $2.9 \%$ of the average calorific value. We avoided excessive localized heating during milling by adding powdered dry ice to the sample as it was

\footnotetext{
${ }^{2}$ For this and all other statements labeled "imprecision," we use the product of the standard deviation of the mean and the appropriate Student $t$ factor at the $95 \%$ confidence level.

${ }^{3}$ For this and all other statements labeled $\% C V$, we use $100 \times s$ average where $s$ is the standard deviation of a single experiment.
}

fed to a Wiley ${ }^{4}$ mill in the final step of size reduction. The larger imprecision of this second result, $2.9 \%$, as compared to the first, $1.1 \%$, is largely due to the fact that identical kilogram-size samples of unprocessed MSW (one of which is then milled) could not be prepared by the procedure we used.

3) We concluded that the calorific values of the gram-size test samples of processed MSW are the same as the calorific values of their kilogram-size parents of unprocessed MSW within $1 \%$. Thus, it is not necessary to combust the entire kilogram-size increment. However, the gram-size test samples must be prepared properly. In particular, the particle size of each increment should be reduced in such a way as to avoid localized heating and the resulting processed MSW should be sampled to obtain gram-size test samples in such a way as to avoid the effects of segregation. Such effects had been observed when coning and quartering were used to obtain gram-size test samples (see reference [1]).

\section{Experimental Apparatus}

The flow calorimeter consists of two basic parts: a constant temperature jacket and the calorimeter proper. The latter contains the sample combustor. Heat liberated in the combustion reaction is transferred to the stirred water in the calorimeter vessel, the outermost container of the calorimeter proper. The rise in temperature of the calorimeter water, after correction for heat exchange with the jacket, is proportional to the enthalpy of combustion of the sample. The calorimeter is calibrated by determining the temperature rise produced by combustion of a known mass of homogeneous, microcrystalline cellulose whose enthalpy of combustion has been measured by bomb calorimetry. (See reference [5], pp. 2226.)

The main features of the flow calorimeter are described in section 3.1. The analysis system used to determine the completeness of combustion (i.e., the $\mathrm{CO}$ concentration in the product gas) and the amount of water vaporized from the calorimeter is described in section 3.2. The data acquisition system used with the calorimeter is described in section 3.3 .

\footnotetext{
${ }^{4}$ Certain facilities, commercial equipment, instruments, or materials are identified in this paper in order to adequately specify the experimental procedure. Such identification does not imply recommendation or endorsement by the National Bureau of Standards, nor does it imply that the materials or equipment are necessarily the best available for the purpose.
} 


\subsection{Description of the Multi-kilogram Capacity Flow Calorimeter}

A cross-section of the entire flow calorimeter is shown in figure 1. The important dimensions and calorimeter properties are listed in table 1. All metal components are fabricated from type 316 stainless steel.

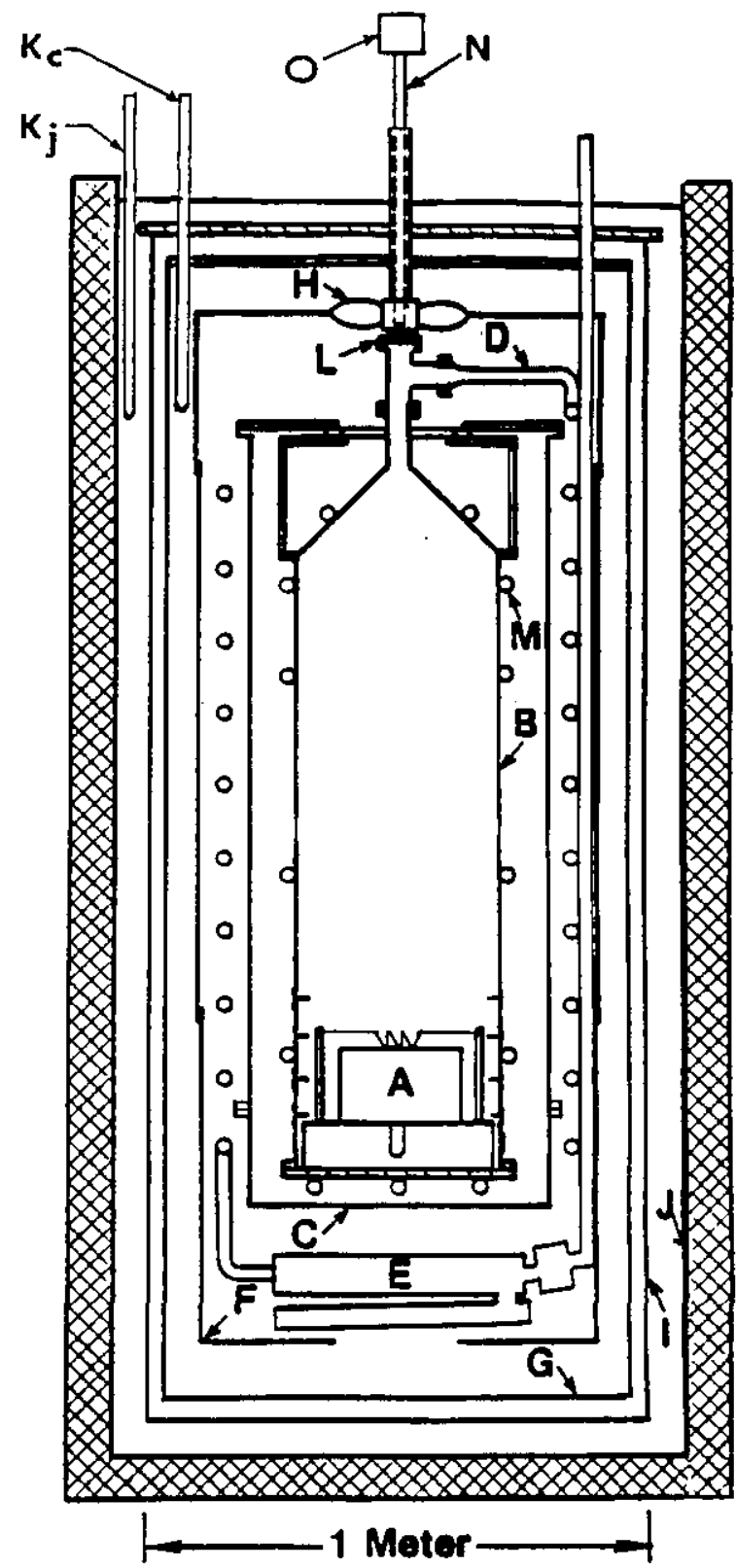

Figure 1-Diagram of the multi-kilogram capacity flow calorimeter. A denotes the sample pellet, $\mathbf{B}$ the combustor, $\mathbf{C}$ the combustor enclosure, $\mathrm{D}$ the heat exchange coil, $\mathrm{E}$ the collector, $F$ the flow shield, $G$ the calorimeter vessel, $H$ the stirrer, I the submarine vessel, $J$ the jacket, $\mathbf{K}_{c}$ the inner quartz oscillator thermometer for the calorimeter water, $\mathbf{K}_{j}$ the outer quartz oscillator thermometer for the jacket water, $\mathrm{L}$ the window, $\mathrm{M}$ the quick-cool heat exchange tube, $\mathrm{N}$ the boroscope, and $\mathrm{O}$ the TV camera.
The cylindrical compressed sample pellet (nominally $23 \mathrm{~cm}$ diameter and $15 \mathrm{~cm}$ high), $A$, is located inside and near the bottom of the combustor, B. A detailed cross-section of the sample and neighboring parts of the combustor is given in figure 2. Referring to that figure, the pellet, $\mathrm{A}$, is supported on a parallel array of nine horizontal, alumina rods, $\mathrm{r}$, (6 $\mathrm{mm}$ diameter) resting in notches cut in the top edge of a cup-shaped ash pan, p. The ash pan, which retains the residual ash from the burned MSW, sits on the base plate, b, that forms the bottom of the combustor. An iron fuse wire, $\mathrm{f}$, having a four turn coil touching the top of the sample at the center, is stretched horizontally between two vertical electrodes of which one, $e$, is shown. The electrodes pass through open-ended tubes of which one, $t$, is shown. The tubes are welded into the bottom of the ash pan. The sample is ignited by passage of electrical current through the fuse wire.

Flowing oxygen is supplied to the sample by an array of nozzles arranged in five horizontal tiers, $o_{1}$ through $\mathrm{O}_{5}\left(\mathrm{O}_{5}\right.$ is not shown in fig. 2). Each tier contains six symmetrically spaced nozzles located in the combustor wall. The bottom tier, $o_{1}$, supplies

Table 1. Specifications for the multi-kilogram capacity flow calorimeter.

\begin{tabular}{lcccc}
\hline Component & \multicolumn{4}{c}{ Dimensions } \\
& $\begin{array}{l}\text { Height } \\
(\mathrm{cm})\end{array}$ & $\begin{array}{c}\text { Outside } \\
\text { Diameter } \\
(\mathrm{cm})\end{array}$ & $\begin{array}{c}\text { Wall } \\
\text { Thickness } \\
(\mathrm{mm})\end{array}$ & $\begin{array}{c}\text { Total } \\
\text { Mass } \\
(\mathrm{kg})\end{array}$ \\
\hline Combustor & 155 & 41 & 3.2 & 74 \\
$\begin{array}{l}\text { Combustor } \\
\text { Enclosure }\end{array}$ & 163 & 61 & 4.8 & 196 \\
$\begin{array}{l}\text { Flow Shield } \\
\text { Calorimeter }\end{array}$ & 213 & 79 & 1.6 & 73 \\
$\begin{array}{l}\text { Vessel } \\
\begin{array}{l}\text { Submarine } \\
\text { Vessel }\end{array}\end{array}$ & 246 & 91 & 4.8 & 390 \\
& & 97 & 4.8 & 454 \\
\end{tabular}

Additional Specifications

$\begin{array}{lll}\text { Calorimeter water } & 980 \text { liters } \\ \text { Jacket water } & 2160 \text { liters } \\ \begin{array}{l}\text { Heat capacity of } \\ \text { calorimeter }\end{array} & 0.4 \mathrm{MJ} / \mathrm{K} \text { (assembly) } \\ & \frac{4.1 \mathrm{MJ} / \mathrm{K}}{4.5 \mathrm{MJ} / \mathrm{K}} \text { (water) } \\ & 4.5 \text { total) }\end{array}$


Figure 2-Cross section of the sample and neighboring parts of the combustor (to scale; pellet height is $15 \mathrm{~cm}$ ). A denotes the pellet, $b$ the combustor base plate, $c_{1}$ through $c_{4}$ the oxygen preheat coils, $d_{1}$ through $d_{4}$ the oxygen distribution rings, e one of the two electrodes, $f$ the iron fuse wire, $M$ the helical tube of the quick-cool heat exchange system, $o_{1}$ through $o_{4}$ the oxygen inlet nozzles, $p$ the ash pan, $r$ the alumina support rods, $t$ an open tube in the ash pan, and $w$ the wall of the combustor. A and $\mathbf{M}$ designate the same components in figures 1 and 2 .

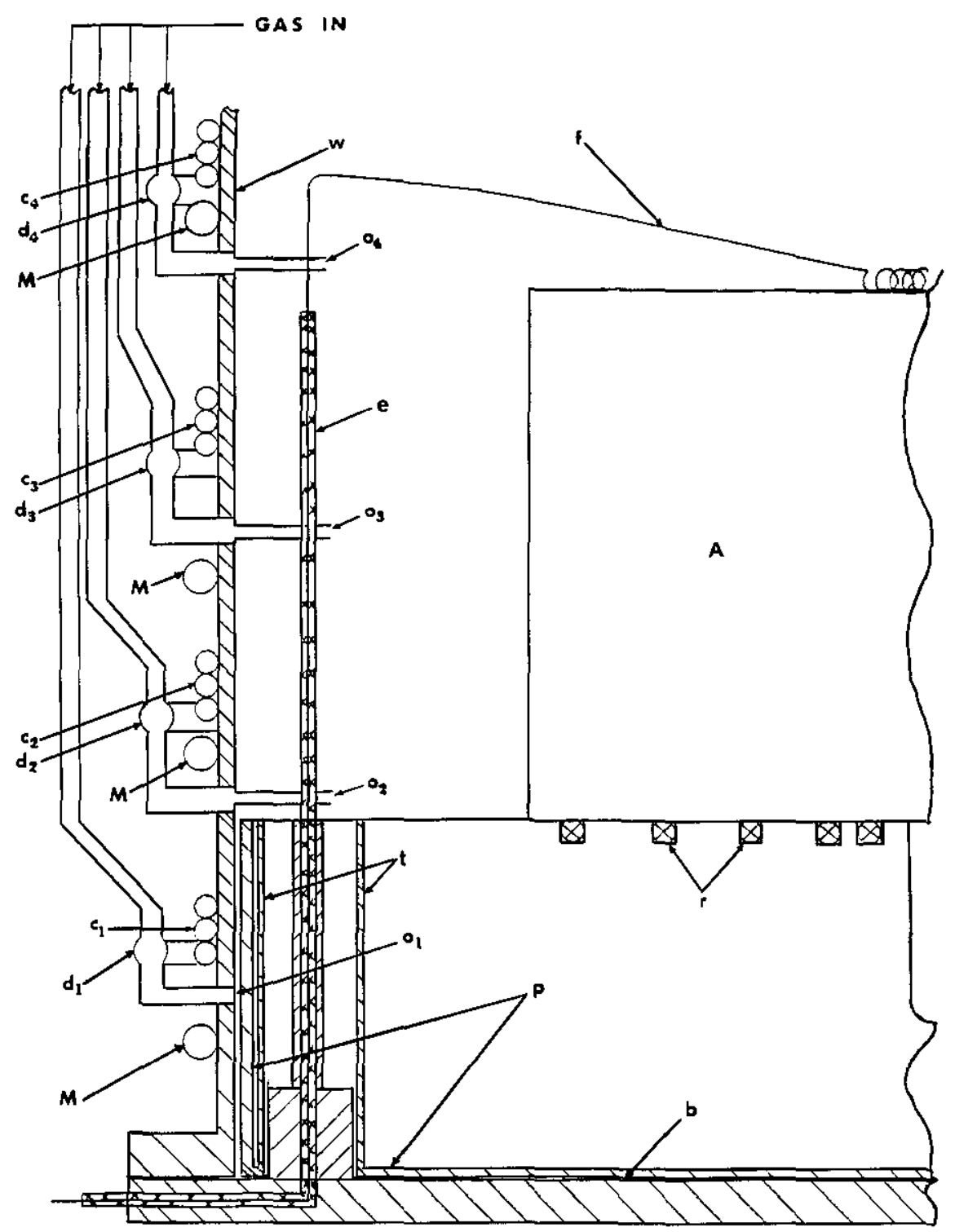

oxygen through slots in the ash pan as a diffuse stream that is directed at the bottom of the sample. The other tiers supply narrow jets of oxygen that are either directed at the sides of the sample or into the space above the sample. The oxygen inlet line for each tier feeds a distribution ring, $d_{1}$ through $d_{5}$, ( $d_{s}$ is not shown in fig. 2) which supplies the oxygen to the nozzles of that tier. The oxygen entering the distribution rings of the top four tiers is first preheated by passing it through separate sets of three coils, $c_{1}$ through $c_{5}$, $\left(c_{5}\right.$ is not shown in fig. 2$)$ wound around and welded to the outside of the combustor wall, w.

Referring back to figure 1 , the combustor, B, is surrounded by a concentric cylindrical vessel, the combustor enclosure, C. The space between the combustor and the combustor enclosure contains the tubes that supply oxygen to the combustor, leads for the thermocouples to monitor the temperature of the oxygen as it enters each distribution ring and the temperature at various locations on the combustor wall, and the fuse ignition leads. The space also contains the helical tube, $M$, of the quick-cool heat-exchange system (the tube is welded to the outside of the combustor with a pitch as shown in fig. 2), and argon gas to reduce oxidation of these components.

A submersible pump circulates the calorimeter water through the quick-cool tube, $\mathbf{M}$, before and after the combustion reaction to ensure that the combustor wall and calorimeter water are at the same temperature during drift periods. Prior to ig- 
nition, the quick-cool tube is purged of water. The relatively poor heat transfer between the combustor enclosure and the combustor allows the temperature of the walls of the combustor to rise during the combustion and, thus, promotes the combustion of any soot deposited on the cold combustor walls during the initial stage of the sample combustion.

The product gas leaves the top of the combustor and then passes downward through a heat-exchange coil, $\mathrm{D}$, having 10 turns. The product gas then passes through the collector, E, through the exit tube, and, finally, out of the calorimeter. The heat exchange coil transfers heat from the product gas to the calorimeter water contained in the space bounded by $\mathrm{C}$ and $\mathrm{G}$. Most of the water formed in the combustion reaction condenses and is trapped in the collector, E. An enlarged cross-section of the collector, E, is shown in figure 3. Referring to that figure, the product gas enters the larger, upper, toroidal tube, $v_{1}$, from the 10-turn heat-exchange coil. Condensate drains from $\mathrm{v}_{1}$ into a second, smaller, lower, toroidal tube, $v_{2}$. The product gas then leaves the collector and passes through glass wool backed by a screen, $s$, in the cylindrical stuffing box, w. The wool reduces the entrainment of condensate droplets in the product gas. Auxiliary dry dilution oxygen is added through a port, $i$, in the stuffing box to reduce the partial pressure of water vapor in the product gas so that it will not condense after the product gas is cooled to room temperature.

Referring back to figure 1 , the calorimeter water is directed past the heat exchange coil by means of the flow shield, F. Water is drawn through the bottom port $(20 \mathrm{~cm}$ diameter) in the flow shield, past the heat exchange coil, D, and is mixed by blades of the stirrer, $\mathrm{H}$, mounted in the top port of the shield. Water returns to the bottom port via the annular space between the calorimeter vessel, $G$, and flow shield at a water flow rate of about 180 $\mathrm{L} / \mathrm{min}$. Thus, the calorimeter water makes a complete circuit of the flow path about every $5.4 \mathrm{~min}$.

The calorimeter vessel is surrounded by the concentric cylindrical submarine vessel, I, which constitutes the innermost portion (i.e., surface) of the constant temperature jacket. The "interspace" between the vessels, which is $2.5 \mathrm{~cm}$ wide, is filled with air which serves to reduce the rate of heat exchange between the jacket and the calorimeter proper. (The top interspace which is between the lids of the calorimeter and the submarine is $5 \mathrm{~cm}$.) The submarine vessel is submerged in the stirred water of the jacket, J. The jacket is a fiberglass covered container whose walls consist of a steel framework covered by polyurethane foam.

The calorimeter water temperature is monitored by the inner quartz oscillator thermometer, $\mathrm{K}_{\mathrm{c}}$. The outer quartz oscillator thermometer, $\mathrm{K}_{\mathrm{j}}$, monitors the temperature of the jacket water. Temperatures of the inlet oxygen supply and the product gas are monitored with thermocouple probes whose junctions are located in the gas tubes at the level of the calorimeter vessel lid. The temperatures of the calorimeter water at the top and bottom of the flow shield are monitored to measure the nonuniformity in the calorimeter water temperature. The measurement junctions of the two separate thermocouples are placed between the calorimeter vessel and the top and bottom edges, respectively, of the flow shield.

The combustion reaction is monitored visually through the window, L, sealed to the inline port of a tee in the product gas line at the top of the com-

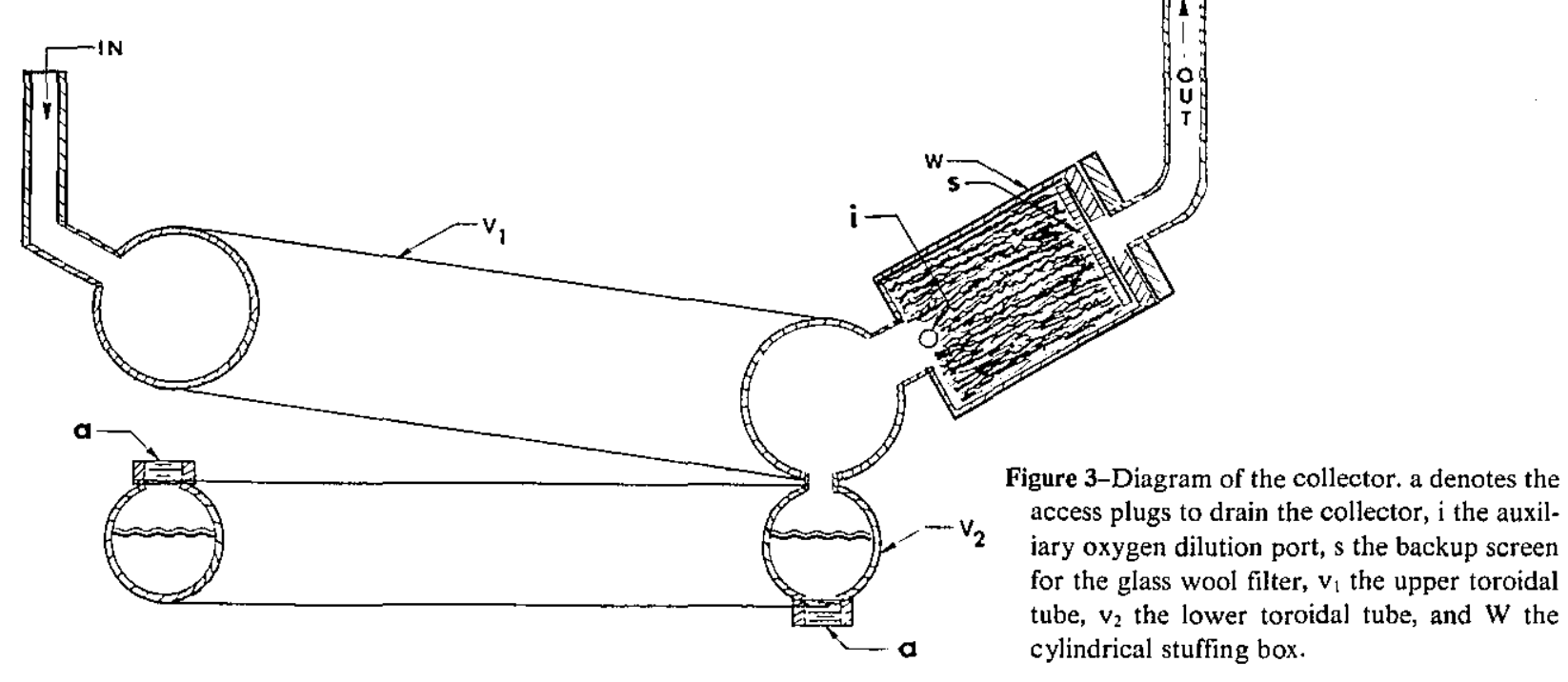


bustor and through the adjacent second window sealed to the end of the hollow stirrer shaft in the water. The boroscope, $\mathrm{N}$, which extends downward through the hollow stirrer shaft, is used to view the combustion. The boroscope is attached to a TV camera, $O$, which is connected to a video monitor-recording system. Oxygen is supplied to the underside of the viewing window to prevent condensation of water on the window during the combustion.

Oxygen is supplied to the calorimeter from six independent sources, each consisting of one or more standard 6200 liter (STP) oxygen tanks that is equipped with reducing and shut-off valves. The oxygen flow from each source is monitored with a mass flow controller. Flowmeters are intercompared by connecting them in parallel and series in appropriate combinations. Two of the meters were calibrated at NBS with an uncertainty of $0.5 \%$.

To simplify the assembly and disassembly of the calorimeter, all the components inside the calorimeter vessel were designed to be suspended from the calorimeter vessel lid and this lid was, in turn, suspended from (beneath) the submarine lid. Motors for the calorimeter stirrer and quick-cool water pump, the boroscope, and the TV camera are mounted in a lifting frame attached to the top of the submarine vessel. The calorimeter plus the submarine vessel at various stages of assembly are raised and lowered with a gantry crane whose hoist chain is connected to the top of the lifting frame. The empty submarine vessel is restrained against the buoyant force of the jacket water by three chains connected between the vessel and eyebolts anchored to the bottom of the jacket. When the calorimeter vessel is unbolted from its lid and the internal calorimeter components are withdrawn, the calorimeter vessel is nested inside the submarine vessel on a removable, concentric collar placed between the top flange of the calorimeter vessel and the top flange of the submarine vessel.

Three features of the calorimeter hardware are significant. First, holes where tubes (containing thermocouple leads, electrical leads, supply oxygen, or product gas) pass through the various lids (e.g., submarine vessel, calorimeter vessel, or combustor enclosure) are closed with stationary O-ring seals. Motor drive shafts pass through chimneys in the submarine vessel lid and rotary O-ring seals in the calorimeter vessel lid. The rotary seals reduce evaporative loss of the calorimeter water when it is warmer than the submarine vessel.

Second, to reduce leakage of gas between the inside of the combustor and the gas space between the combustor and the combustor enclosure, the combustor base plate is sealed to the rest of the combustor by a gasket that is a composite of graphite and 316 stainless steel. The combustor enclosure itself is made gas-tight by use of Viton $\mathrm{O}$ ring seals. Electrical leads to the inside of the combustor enclosure are hermetically sealed with epoxy cement in their feed-through tubes.

Third, the reference junctions of all thermocouples on the combustor, in the calorimeter water, and in the flow lines (exclusive of those in the product gas analysis train) are mounted on an aluminum reference block which is inside a hermetic chamber that connects by tubes to the combustor enclosure. These tubes feed the thermocouple leads from the combustor through the various lids and support the block and chamber above the level of the jacket water. The hermetic seal in the chamber completes the gas-tight seal of the combustor enclosure. The temperature of the block is allowed to float (i.e., it is not controlled) but its temperature is monitored by the calorimeter water thermocouple whose junction is located at the top edge of the flow shield and is close to the inner quartz oscillator thermometer, $\mathbf{K}_{\mathrm{c}}$. During an experiment, the block temperature changes by less than $0.1{ }^{\circ} \mathrm{C}$.

\subsection{Analysis Systems}

After leaving the submarine vessel, the product gas passes through a mixing chamber. Just beyond the chamber, a portion of the gas is continuously withdrawn for analysis; the product gas at this point is at or near room temperature. A block diagram of the analysis system is shown in figure 4. Part of the gas withdrawn for analysis is dried and passed at a flow rate of about $2 \mathrm{~L} / \mathrm{min}$ through dedicated infrared detectors for $\mathrm{CO}$ and $\mathrm{CO}_{2}$. A second part of the gas is passed at a flow rate of about $2 \mathrm{~L} / \mathrm{min}$ through a cooled-mirror type of automatic dew point detector to monitor water vapor. The remaining part of the analysis gas is passed at a flow rate of about $5 \mathrm{~L} / \mathrm{min}$ through a scanning infrared detector used to monitor various trace components (such as hydrocarbons, $\mathrm{SO}_{2}$, $\mathrm{HCl}$, etc.). These detectors in combination with three manometers and four thermocouples (to monitor gas temperatures) are used to determine the composition of the product gas.

\subsection{Data Aquisition Equipment}

The enormous amount of data generated during each experiment was monitored and recorded using the system shown schematically in figure 5 . Because of the delays associated with displaying and 
Figure 4-Block diagram of the product gas analysis system.

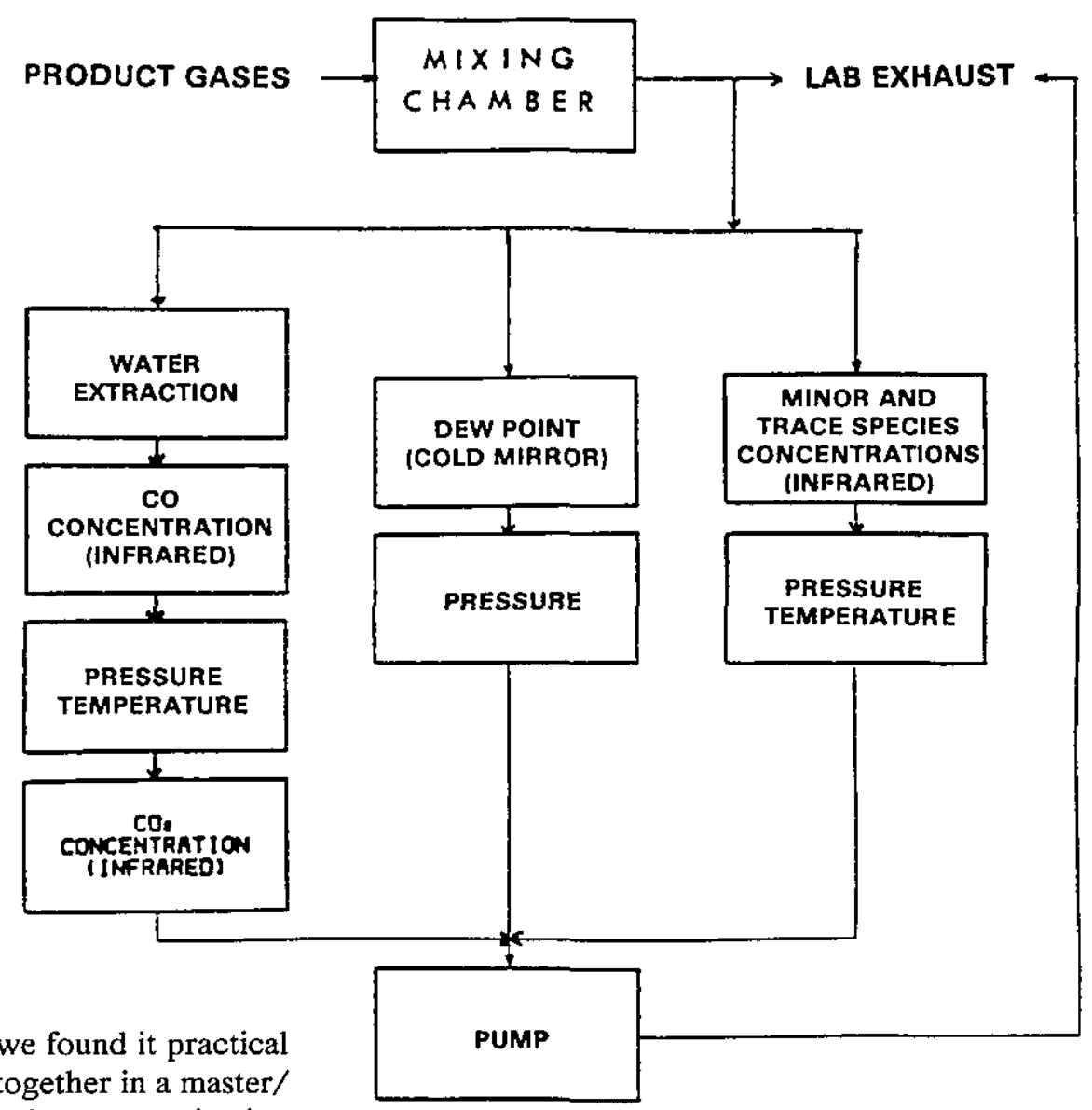

of water lost from the calorimeter, the signal from each of these detectors was recorded at least three times every minute.

For the experiments described in this paper, it was convenient to record the ouput signal of the scanning infrared detector on a strip chart. No significant energy loss was associated with very small concentrations of the trace components in the product gas.

\section{Description of an Experiment and Typical Results}

The time frame, operating parameters, and significant outputs of a typical experiment are described in section 4.1. An idea of the time frame of preparation for an experiment and some of the details of the assembly of the calorimeter are given as the large size of the calorimeter magnifies time and manpower needs and produces problems in otherwise trivial operations. A summary of data reduction procedures is given in section 4.2. Typical calculated results for calibration and "unknown" experiments are given in section 4.3. 


\section{Journal of Research of the National Bureau of Standards}

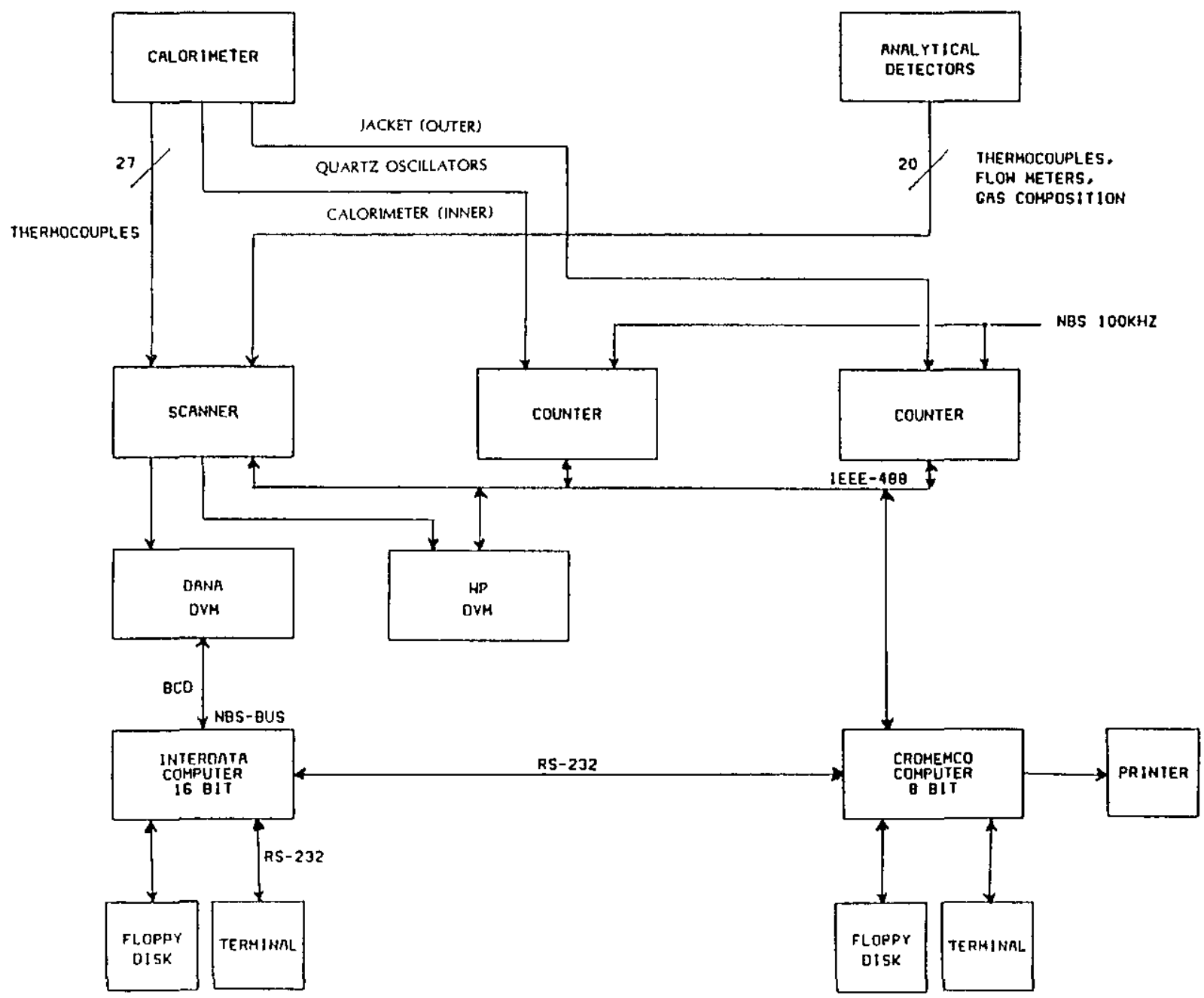

Figure 5-Block diagram of the data acquisition system.

\subsection{Experimental Procedure}

On the day prior to the experiment, the sample pellet is prepared and the components inside of the calorimeter vessel are assembled.

On the day of the experiment, the calorimeter water is heated (or cooled) in a separate holding tank to a temperature within $\pm 0.3^{\circ} \mathrm{C}$ of that of the jacket water which is within $\pm 1^{\circ} \mathrm{C}$ of room temperature $\left(22^{\circ} \mathrm{C}\right)$. A fixed volume of water from the holding tank is added to partially fill the empty calorimeter vessel; the volume is the maximum amount that experience shows will not splash or overflow as the internal components of the calorimeter are lowered into the calorimeter vessel. The volume is determined with a jig, shown in figure 6 , which has a filling cup, fc, having a conical bottom and an overflow hole, oh, located in its side. The upper supports, sp, are bolted to the top surface of the calorimeter vessel, G, so that the cup is inside ( $1.3 \mathrm{~cm}$ radial clearance) and at a fixed distance (about $75 \mathrm{~cm}$ ) below the top flange of the vessel. After leveling the top surface of the calorimeter vessel to within \pm 20 seconds of arc using the spirit level, I, water is added until it just overflows into the inside of the filling cup. After the jig is removed, the internal components of the calorimeter are gently lowered into the vessel. The calorimeter vessel and submarine vessel are bolted to their respective lids. After lowering the entire assembly into the jacket water, the final 20 liters (fixed for all runs) of water needed to fill the calorimeter vessel to within $0.3 \mathrm{~cm}$ of its lid are added.

The stirrers for the calorimeter and jacket water are turned on, and the calorimeter water temperature is monitored for one hour after the drift rate becomes constant. During this time interval, the calorimeter water is pumped through the quickcool heat exchange tube on the combustor wall. 


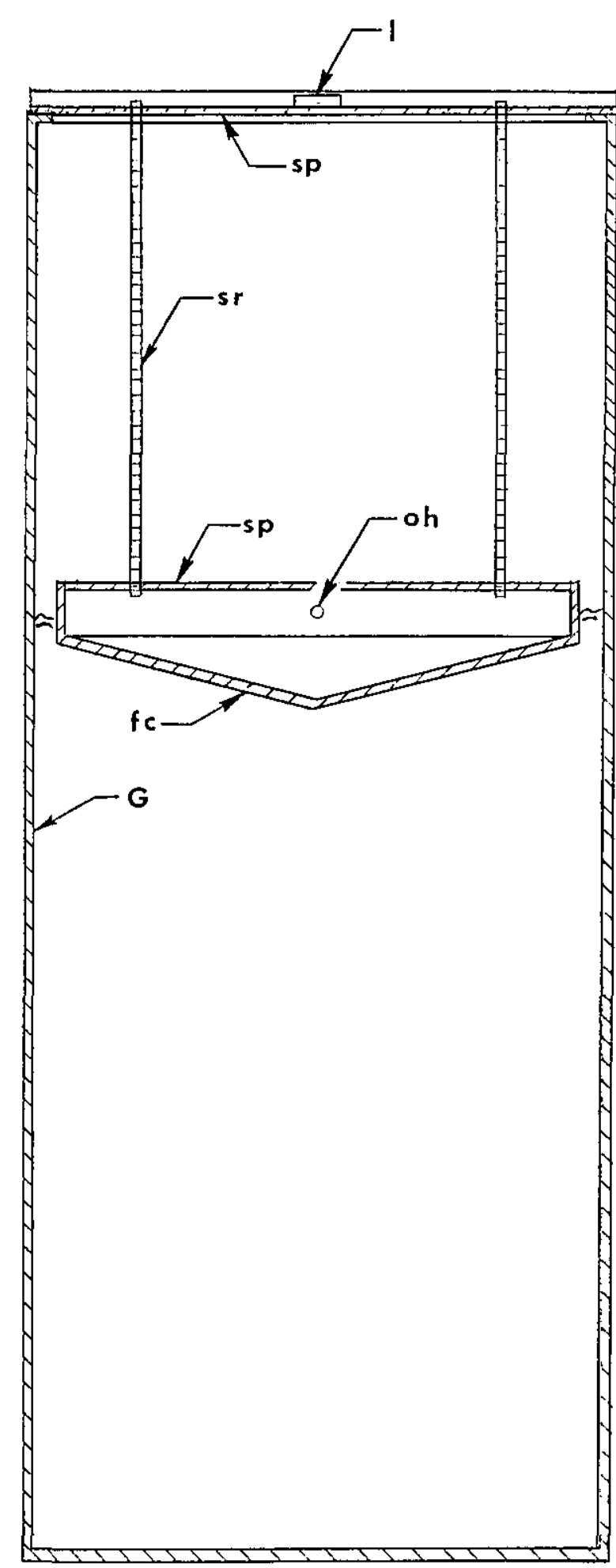

Figure 6-Diagram of the calorimeter water loading jig. fc denotes the filling jig cup (cup-shaped pan), $G$ the calorimeter vessel, I the spirit level, oh the overflow hole in filling jig cup, sp the filling jig cup supports, and sr the filling cup spacing rods.
The last 30 minutes of the time interval is the initial drift period. ${ }^{5}$

Twelve and one-half minutes prior to ignition, the flow of water to the quick-cool heat exchange tube is diverted to the calorimeter vessel and the water remaining in the quick-cool tube is flushed out with compressed air. The combustor is then flushed with oxygen for 10 minutes at a flow rate of $30 \mathrm{~L} / \mathrm{min}$. (Flow volumes are expressed in liters at $0{ }^{\circ} \mathrm{C}$ and $101.325 \mathrm{kPa}$.) Two and one half minutes prior to ignition, the flow rates are increased to the levels used during combustion when oxygen is supplied at constant rates of $150 \mathrm{~L} / \mathrm{min}$ to the combustor, $10 \mathrm{~L} / \mathrm{min}$ to the viewing window, and $110 \mathrm{~L} / \mathrm{min}$ to the dilution port at the collector exit for a total flow of $270 \mathrm{~L} / \mathrm{min}$.

Sample burning times were approximately 32 minutes for pellets of unprocessed MSW or microcrystalline cellulose, and about 42 minutes for processed MSW. The $\mathrm{CO}_{2}, \mathrm{H}_{2} \mathrm{O}$, and $\mathrm{CO}$ concentrations in the product gas during the combustion of a processed MSW pellet are shown in figure 7. The peak and average $\mathrm{CO}_{2}$ concentrations over the first 10 minutes of the combustion are 52 and 38 mole percent, respectively. The latter concentration corresponds to an inlet oxygen flow rate of about 2.5 times stoichiometry.

The $\mathrm{H}_{2} \mathrm{O}$ concentration reaches a maximum of 2.4 mole percent (i.e., dew point of $16^{\circ} \mathrm{C}$ ) at 30 minutes; the abrupt fall in concentration at $42 \mathrm{~min}$ utes indicates that the combustor has been completely flushed of product gas. The inlet oxygen flow rate is maintained at the same level as during the combustion reaction for another 10 minutes to assist in the "cool off" of the combustor. The total inlet oxygen flow rate is then reduced to about 20 $\mathrm{L} / \mathrm{min}$ to prevent excessive evaporation of water from the collector. This flow, which is supplied only to the combustor, maintains a positive pressure in the combustor with respect to the collector and thus prevents backflow of condensate into the combustor. The inlet oxygen flow is stopped prior to the beginning of the final drift period.

The occurrence of two peaks in the $\mathrm{CO}$ concentration versus time is typical of all the MSW samples. In figure 7, the initial and final maximum $\mathrm{CO}$ concentrations are 0.016 and 0.027 mole percent, respectively. The initial peak always occurs near the time of the maximum rate of production of $\mathrm{CO}_{2}$; the final peak occurs near the end of the vi-

\footnotetext{
s Strictly, the initial drift period ends 12.5 minutes prior to ignition when the oxygen supply used to flush out the calorimeter is turned on. In practice, the error incurred in $\Delta T_{\mathrm{c}}$ of eq (3) and $\Delta H_{\mathrm{g}}$ of eq (1) by assuming that the initial drift period ends at the time of ignition has a negligible effect on our results.
} 


\section{Journal of Research of the National Bureau of Standards}

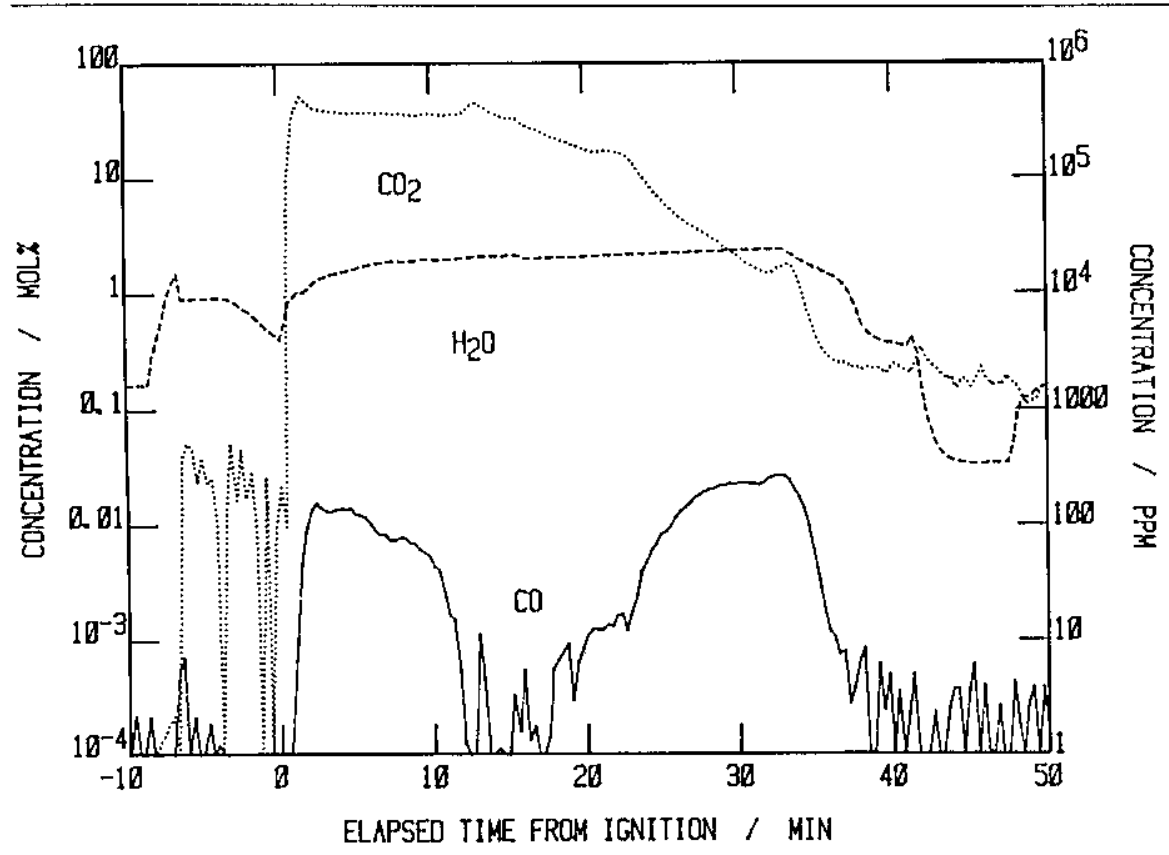

Figure 7-A plot of the concentrations of gaseous carbon dioxide, water, and carbon monxide as a function of time for a typical experiment on MSW.

sual burning when the ash tends to inhibit the combustion. The average $\mathrm{CO}$ concentration in a cellulose combustion is about a factor of 50 smaller than that shown in figure 7.

The temperature of the product gas as it leaves the top of the combustor rises to about $400^{\circ} \mathrm{C}$ within the first minute of the combustion, gradually increases to a maximum of about $450^{\circ} \mathrm{C}$ between 12 and 15 minutes after ignition, and then decays exponentially thereafter. The temperatures of the combustor wall and of the oxygen gas as it leaves the preheat coils increase more gradually to their peak values at about the same time and decay similarly. The peak values of the temperatures of the wall and adjacent preheat coils within $25 \mathrm{~cm}$ of the ash pan are between 600 and $700^{\circ} \mathrm{C}$.

When the temperature of the combustor base plate has decreased to $220^{\circ} \mathrm{C}$, the calorimeter water is again circulated through the quick-cool heat exchange tube. This safely removes the appreciable excess heat stored in the combustor. A plot of the calorimeter water temperature versus elapsed time for the experiment that corresponds to figure 7 is shown by the solid line of figure 8 . When the quick-cool system was activated, which occurred at an elapsed time of 65 minutes, the temperature of

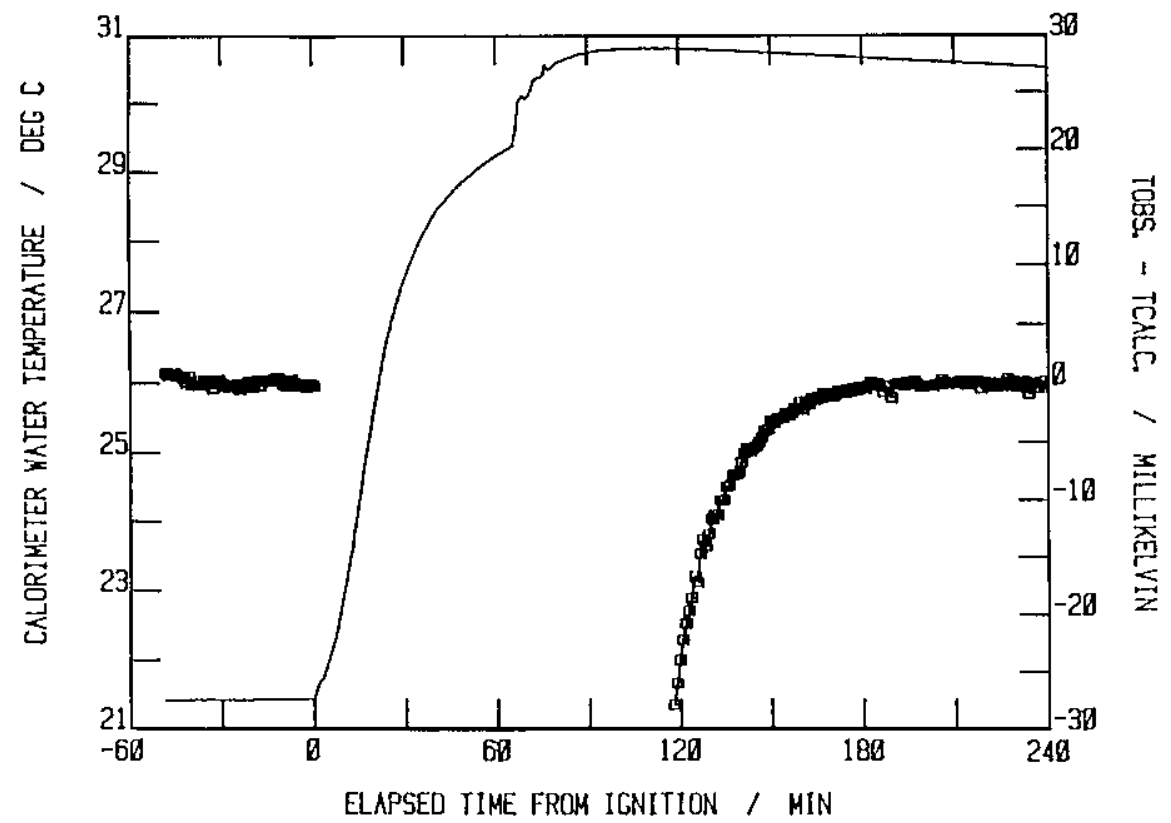

Figure 8-A plot of the observed temperature rise of the calorimeter water plus the deviation of the observed data points from the smooth curve fit to the data during the drift periods. The effect of activation of the quick-cool system is seen at about 65 minutes. 


\section{Journal of Research of the National Bureau of Standards}

the calorimeter water has increased $8{ }^{\circ} \mathrm{C}$. During the next 10 minutes, when most of the excess heat was removed, the temperature of the calorimeter water increased by another $1.2^{\circ} \mathrm{C}$. The latter increase indicated that $13 \%$ of the total heat liberated by the combustion reaction was stored in the walls and base plate of the combustor.

After the drift rate of the calorimeter water attains a steady value (which occurs about three hours after ignition) the inlet oxygen flow is stopped and temperature readings are made for an additional hour. At this point, the combustor and calorimeter water are at the same temperature to within $0.1{ }^{\circ} \mathrm{C}$. The last 30 minutes of this time interval corresponds to the final drift period.

Before assembly and after disassembly of the calorimeter, the ash pan with its contents was weighed to determine the mass of ash formed in the combustion experiment. Similarly, the collector was weighed to determine the mass of water which was formed in the combustion reaction and remained in the calorimeter. The ash was milled, homogenized, and a sample was analyzed for moisture, carbon, and hydrogen content. The condensate in the collector was analyzed for total $\mathrm{H}^{+}$, $\mathrm{Cl}^{-}, \mathrm{SO}_{4}{ }^{-}$, and $\mathrm{NO}_{3}{ }^{-}$contents.

\subsection{Data Reduction}

Results are calculated using a format adopted from the work of Prosen et al. [6]. The basic measurement equation is:

$$
\begin{aligned}
E \Delta T_{\mathrm{c}} & =-\left(M \cdot \Delta \vec{H}_{\mathrm{st}}+\Delta H_{\mathrm{v}}+\Delta H_{\mathrm{g}}+\Delta H_{\mathrm{ic}}\right. \\
& \left.+\Delta H_{\mathrm{ig}}+\Delta H_{\mathrm{x}}\right)+W .
\end{aligned}
$$

In eq (1), $E$ is the energy equivalent of the calorimeter. The parameter $\Delta T_{\mathrm{c}}$ is the corrected temperature rise of the calorimeter. The parameter $M$ is the mass of the sample. The parameter $\Delta \bar{H}_{\mathrm{st}}$ is the enthalpy change per unit mass of sample for an assumed standard reaction in which all reactants and products are at some selected isothermal process temperature, $T_{\mathrm{h}}$, and pressure of $101.325 \mathrm{MPa}$ (1 atm). The remaining five terms on the right of eq (1) account for the conditions of a particular experiment. They are as follows: $\Delta H_{v}$ is the correction for the vaporization of water formed in the combustor that is lost from the calorimeter (i.e., in product gases). The parameter $\Delta H_{\mathrm{g}}$ is the correction for the net heat transport of gas entering and leaving the calorimeter at some temperature other than $T_{\mathrm{k}}$. The parameter $\Delta H_{\mathrm{ic}}$ is the correction for incomplete combustion of the combustible fraction of the sample to the assumed products of the stan- dard reaction. The parameter $\Delta H_{\mathrm{ig}}$ is the correction for the enthalpy of oxidation of the fuse. The parameter $\Delta H_{\mathrm{x}}$ is the correction for deviations of the conditions of the experiment from a constant pressure process at $1 \mathrm{~atm}$ assuming all gases are perfect and the kinetic energy of the flowing gases is negligible. The parameter $W$ is the electrical work required to heat the fuse wire.

The corrected temperature rise, $\Delta T_{\mathrm{c}}$, is evaluated in the conventional manner (see reference [7], p. 2-15). The drift period equation, eq (2) below, is fitted to the observed calorimeter water temperature, $T_{1}(t)$, versus time, $t$, data of the initial and final drift periods using a computer program given in reference [8].

$$
d T_{1}(t) / d t=k\left(T_{\infty}-T_{1}(t)\right)
$$

In the application of eq (2), $k$, the cooling constant, and $T_{\infty}$, the convergence temperature, are assumed to be constant and identical for both drift periods. The data points in figure 8 are the deviations of $T_{1}(t)$ from the integral form of eq (2). (The ordinate scale is -30 to $+30 \mathrm{mK}$.) The set of data points on the right of the figure indicates that the final drift period begins about 150 to 180 minutes after ignition. The corrected temperature rise is given by eq (3).

$$
\Delta T_{\mathrm{c}}=T_{1}\left(t_{\mathrm{f}}\right)-T_{1}\left(t_{\mathrm{i}}\right)-k \int_{\mathrm{t}_{\mathrm{i}}}^{\mathrm{t}_{\mathrm{f}}}\left(T_{\infty}-T_{\mathrm{l}}(t)\right) \mathrm{d} t
$$

In eq (3), $t_{\mathrm{i}}$ and $t_{\mathrm{f}}$ are times at the end of the initial drift period (ignition of the sample, see footnote 5) and at the beginning of the final drift period, respectively. $T_{1}\left(t_{i}\right)$ and $T_{1}\left(t_{t}\right)$ are called the initial and final temperatures of the calorimeter, respectively. The observed temperature rise is $T_{1}\left(t_{f}\right)-T_{1}\left(t_{\mathrm{i}}\right)$, and the correction for the heat exchange with the surroundings is the last term on the right of eq (3). The sample size was chosen so that the observed temperature rise was between 6.0 and $9.5^{\circ} \mathrm{C}$ for all experiments. The heat exchange correction amounted to about $4 \%$ of the observed temperature rise. The cooling constant, $k$, is typically about $3 \times 10^{-4} \mathrm{~min}^{-1}$ and $T_{\infty}$ is about $22^{\circ} \mathrm{C}$ (near room temperature).

Whether the energy equivalent, $E$, refers to the calorimeter when the products or reactants are present is, in principle, determined by the selection of the isothermal process temperature, $T_{\mathrm{h}}$ (see reference [7], p. 2-6 and reference [9]). For this calorimeter, the difference in $E$ for $T_{\mathrm{h}}=T_{\mathrm{l}}\left(t_{\mathrm{i}}\right)$ or $T_{\mathrm{h}}=T_{\mathrm{i}}\left(t_{\mathrm{f}}\right)$ is negligible. In our work, it is convenient to take $T_{\mathrm{h}}$ equal to the initial calorimeter tem- 


\section{Journal of Research of the National Bureau of Standards}

perature $T_{1}\left(t_{\mathrm{i}}\right)$ so that $E$ refers to the calorimeter after the combustion reaction.

Since the value of $E$ varies with the amount of ash remaining in the combustor (e.g., none in a calibration reaction) and water remaining in the collector, these variations are taken into account by adding the appropriate heat capacity difference, denoted by $\delta E$, to the value of $E$ calculated from each calibration run to obtain $E_{\mathrm{s}}$. The parameter $E_{\mathrm{s}}$ is the energy equivalent under an arbitrary but fixed set of so-called "standard calorimeter conditions." A different but appropriate $\delta E$ is subtracted from the average $E_{\mathrm{s}}$ to obtain the energy equivalent for each MSW combustion experiment.

For the calibration reaction with microcystalline cellulose, the products of the standard reaction are taken to be $\mathrm{CO}_{2}(\mathrm{~g})$ and $\mathrm{H}_{2} \mathrm{O}(\mathrm{l})$. For the combustible fraction of MSW, that contains primarily $\mathrm{C}, \mathrm{H}, \mathrm{O}$, $\mathrm{N}, \mathrm{S}$, and $\mathrm{Cl}$, the products of the standard reaction are taken as $\mathrm{CO}_{2}(\mathrm{~g}), \mathrm{H}_{2} \mathrm{O}(\mathrm{l}), \mathrm{N}_{2}(\mathrm{~g}), \mathrm{SO}_{2}(\mathrm{~g})$ and $\mathrm{HCl}(\mathrm{aq})$. (Gases are assumed to be perfect and solutions are assumed to be ideal.) As a consequence, $\Delta H_{\text {ic }}$ contains the corrections for the presence of $\mathrm{CO}(\mathrm{g})$ in the product gases, carbon in the ash, and a correction for the presence of any appreciable $\mathrm{SO}_{4}{ }^{=}$and $\mathrm{NO}_{3}^{-}$in the collector condensate. Separate experiments using a flame ionization detector show that the concentration of gaseous hydrocarbons in the product gas is negligible.
The reactions that correspond to $\Delta \bar{H}_{\mathrm{st}}, \Delta H_{\mathrm{v}}$, and $\Delta H_{\mathrm{g}}$ and the method used to calculate the correction terms in eq (1) are similar (see reference [10]) to those given in reference [3].

\subsection{Typical Results}

Calibration results are given in table 2. The second column is the standard enthalpy of combustion per unit mass of dry cellulose as determined by bomb calorimetry after correction to the value of $T_{\mathrm{h}}$ appropriate to each experiment (i.e., $T_{\mathrm{h}}=T_{1}\left(t_{\mathrm{i}}\right)$ ). The third column is the dry sample mass, $M$. Quantities in columns five through nine were defined in the previous section. The ten are column entries in the correction, $\delta E$, added to the energy equivalent calculated according to eq (1) to obtain the energy equivalent, $E_{\mathrm{s}}$, for standard calorimeter conditions. The standard calorimeter conditions are defined to be: the calorimeter water is $21^{\circ} \mathrm{C}$ immediately after it has been assembled and filled with water, no ash remains in the ash pan, and the mass of condensate remaining in the collector after the reaction is $1215 \mathrm{~g} . E_{\mathrm{s}}$ is given in the last column of table 2 . The average energy equivalent, $E_{\mathrm{s}}$, and the standard deviation of a single measurement are listed at the bottom of the table.

The \% $C V$ of the determination of $E_{\mathrm{s}}, 0.19 \%$, is comparable in magnitude to the $\% C V$ of the 11

Table 2. Calculation of the energy equivalent of the calorimeter.

\begin{tabular}{|c|c|c|c|c|c|c|c|c|c|c|}
\hline Col. & (2) & (3) & (4) & (5) & (6) & (7) & (8) & (9) & $(10)$ & (11) \\
\hline \multirow[t]{2}{*}{$\begin{array}{l}\text { Exp. } \\
\text { No. }\end{array}$} & $-\Delta \tilde{H}_{\mathrm{s} 1}$ & $\begin{array}{c}M \\
\text { (dry) }\end{array}$ & $-M \cdot \Delta H_{\mathrm{st}}$ & $\Delta H_{\mathrm{v}}$ & $\Delta H_{\mathrm{g}}$ & $\begin{array}{l}\Delta H_{\mathrm{ic}} \\
(\mathrm{CO})\end{array}$ & $-\Delta H_{\mathrm{ig}}+W$ & $\Delta T_{\mathrm{c}}$ & $\delta E^{(a)}$ & $E_{\mathrm{s}}^{(\mathrm{b})}$ \\
\hline & $\mathrm{MJ} / \mathrm{kg}$ & $\mathrm{kg}$ & MJ & $\mathrm{MJ}$ & MJ & $\mathrm{MJ}$ & $\mathrm{MJ}$ & $\mathbf{K}$ & $\mathrm{MJ} / \mathrm{K}$ & $\mathrm{MJ} / \mathrm{K}$ \\
\hline 9 & 17.340 & 2.0474 & 35.502 & $(0.217)^{(\mathrm{c})}$ & 0.022 & $(0.000)^{(\mathrm{d})}$ & 0.002 & 7.8652 & -0.0019 & 4.4818 \\
\hline 10 & 17.345 & 2.0429 & 35.434 & $(0.263)^{(\mathrm{c})}$ & -0.012 & $(0.000)^{(\mathrm{d})}$ & do & 7.8447 & -0.0022 & 4.4830 \\
\hline 11 & 17.336 & 2.0659 & 35.814 & 0.285 & 0.048 & 0.000 & do & 7.9514 & 0.0000 & 4.4625 \\
\hline 15 & 17.349 & 2.0859 & 36.188 & 0.203 & -0.042 & 0.001 & & & & \\
\hline 24 & 17.335 & 2.2856 & 39.621 & 0.304 & 0.043 & 0.001 & do & 8.7449 & +0.0001 & 4.4913 \\
\hline 29 & 17.335 & 2.2883 & 39.667 & 0.367 & 0.077 & 0.000 & do & 8.7728 & +0.0003 & 4.4715 \\
\hline 30 & 17.335 & 2.3836 & 41.320 & 0.321 & 0.064 & do & do & 9.1495 & 0.0000 & 4.4742 \\
\hline 35 & 17.337 & 2.3239 & 40.289 & 0.330 & 0.066 & do & do & 8.9142 & -0.0006 & 4.4748 \\
\hline \multirow[t]{3}{*}{36} & 17.336 & 2.4584 & 42.619 & 0.313 & 0.065 & do & do & 9.4261 & -0.0009 & 4.4806 \\
\hline & & & & & & \multicolumn{4}{|c|}{ Average } & 4.4767 \\
\hline & & & & & & \multicolumn{4}{|c|}{ Std. dev. $(\% \mathrm{CV})^{\mathrm{e}}$} & $0.0086(0.19 \%)$ \\
\hline
\end{tabular}

\footnotetext{
(a) Correction for density of water at filling temperature other than $21^{\circ} \mathrm{C}$ and for the difference of the mass of water in the collector from $1215 \mathrm{~g}$; add to $E$ of eq (1) to obtain $E_{\text {s. }}$.

(b) $E_{\mathrm{s}}$ is $E$ at filling temperature of $21^{\circ} \mathrm{C}$ and $1215 \mathrm{~g}$ water in collector after the reaction.

(c) Estimated assuming that the mass of water vaporized is the water formed (as calculated from the empirical formula) minus that remaining in the collector.

(d) $\mathrm{CO}$ detector not functioning properly; $\mathrm{CO}$ assumed to be zero.

(e) Includes variance of mean $\Delta \bar{H}_{\mathrm{st}}$ of cellulose. Without latter, the standard deviation is 0.0084 and $\% \mathrm{CV}$ is $0.19 \%$.
} 


\section{Journal of Research of the National Bureau of Standards}

bomb calorimetric determinations of $-\Delta \bar{H}_{\mathrm{st}}$ of cellulose, $0.15 \%$. For the bomb calorimeter used in the latter measurements, the \% $C V$ for the determination of its energy equivalent by combustion of standard reference material (SRM) benzoic acid was $0.05 \%$. (A state-of-the-art bomb calorimeter has a $\% C V$ of $0.01 \%$ or less for the determination of its energy equivalent by SRM benzoic acid.)

The magnitudes of the average corrections to the calibration data are listed in the third column of table 3. The correction to the observed temperature rise to obtain $\Delta T_{\mathrm{c}}, 4 \%$ of $\Delta T_{\mathrm{c}}$, is the largest correction. The next largest correction is the vaporization correction, $\Delta H_{v}$, which is $0.75 \%$ of $M \cdot \Delta \bar{H}_{\text {st }}$ followed by the gas heat transport correction, $\Delta H_{\mathrm{g}}$ which is about $0.1 \%$ of $M \cdot \Delta \vec{H}_{\mathrm{sc}}$. The correction for $\mathrm{CO}$ is negligible and no ash or residue remained from the combustion reaction.

As indicated in the third row of table 3, an average of $91.0 \%$ of the total water formed in the reac- tion is retained in the collector. The measured total water formed in the combustion reaction was $100.67 \pm 0.42 \%$ (imprecision of the mean of experiments 11 through 36 of table 2) of the water produced by the combustion of cellulose as calculated from its empirical formula of $\mathrm{C}_{5} \mathrm{H}_{10} \mathrm{O}_{5}$ (s). The measured $\mathrm{CO}_{2}$ was $99 \pm 3 \%$ (imprecision of the mean of experiments 11 through 36 of table 2) of the calculated value of $\mathrm{CO}_{2}$.

The results for the enthalpy of combustion of four sets of paired samples of processed MSW are given in table 4. The pairs of samples designated as $E$ and $E^{\prime}$ followed by the same number were prepared in such a way that each sample is "identical" (see reference [5], p. 19-22). The energy equivalent, $E$ in column 3 is the average value of $E_{\mathrm{s}}$ taken from table 3 plus a correction for the variation in filling temperature from $21^{\circ} \mathrm{C}$ and for the difference between the amount of water retained in the collector and ash in the combustor in these experi-

Table 3. Magnitudes of average enthalpy corrections of the basic measurement equation, eq (1).

\begin{tabular}{|c|c|c|c|}
\hline Type of Correction & Symbol Form & Cellulose & Processed MSW \\
\hline Correction for heat exchange & $\left|\left(\Delta T_{\mathrm{c}}-\Delta T_{\mathrm{l}}\right) / \Delta T_{\mathrm{c}}\right|^{\text {(a.t) }}$ & $4.00 \%$ & $3.50 \%$ \\
\hline Vaporization of water & $\left|\Delta H_{\mathrm{v}} / M \cdot \Delta H_{\mathrm{s} 1}\right|$ & $0.75 \%$ & $1.42 \%$ \\
\hline (water in collector/total water) & & $(91.0 \%)$ & $(81.4 \%)$ \\
\hline Gas heat transport correction & $\left|\Delta H_{\mathrm{g}} / \boldsymbol{M} \cdot \Delta \ddot{\boldsymbol{H}}_{\mathrm{st}}\right|$ & $0.12 \%$ & $0.13 \%$ \\
\hline Correction for incomplete combustion & $\left|\Delta H_{\mathrm{ic}} / M, \Delta \vec{H}_{\mathrm{s:}}\right|$ & & \\
\hline $\mathrm{CO}(\mathrm{g})$ & & $0.00 \%$ & $0.13 \%$ \\
\hline carbon in ash & & ${ }_{-}-^{\text {(c) }}$ & $0.03 \%$ \\
\hline $\mathrm{SO}_{4}(\mathrm{aq})$ rather than $\mathrm{SO}_{2}(\mathrm{~g})$ & & - & $0.01 \%$ \\
\hline $\mathrm{NO}_{3}^{-}(\mathrm{aq})$ rather than $\mathrm{N}_{2}(\mathrm{~g})$ & & - & $--\infty$ \\
\hline
\end{tabular}

(a) $\Delta T_{1}$ is the observed temperature rise, $T_{1}\left(t_{0}\right)-T_{1}\left(t_{1}\right)$.

(b) | | indicates absolute value.

(c) no ash formed.

(d) carbon in ash is assumed to be elemental carbon.

Table 4. Calculation of $-\Delta H_{\mathrm{st}}$ for processed MSW samples.

\begin{tabular}{|c|c|c|c|c|c|c|c|c|c|c|c|}
\hline $\begin{array}{l}\text { Column } \\
\text { No. } \\
\text { Sample } \\
\text { No. }\end{array}$ & $\begin{array}{c}(2) \\
\Delta T_{\mathrm{E}} \\
\mathrm{K}\end{array}$ & $\begin{array}{c}E \\
\mathrm{MJ} / \mathrm{K}\end{array}$ & $\begin{array}{c}\text { (4) } \\
E \Delta T_{\mathrm{c}} \\
M \mathrm{~J}\end{array}$ & $\begin{array}{c}\Delta H_{\mathrm{v}} \\
\mathrm{MJ}\end{array}$ & $\begin{array}{r}\Delta H_{\mathrm{g}} \\
\mathbf{M J}\end{array}$ & $\begin{array}{c}\text { (7) } \\
(\mathrm{CO}) \\
\mathrm{MJ}\end{array}$ & $\begin{array}{c}(8) \\
-\Delta H_{\mathrm{iz}} \\
\mathrm{C}(\mathrm{ash})^{(2)} \\
\mathrm{MJ}\end{array}$ & $\begin{array}{c}\text { (9) } \\
\mathrm{SO}_{4}=(\mathrm{aq}) \\
\mathrm{MJ}\end{array}$ & $\begin{array}{c}(10) \\
\underset{\mathrm{MJ}}{\left(\Delta H_{\mathrm{ig}}-W\right)}\end{array}$ & $\begin{array}{c}(11) \\
M(\mathrm{dry}) \\
\mathrm{Kg}\end{array}$ & $\begin{array}{c}\text { (12) } \\
-\Delta \bar{H} \mathrm{st} \\
\mathrm{MJ} / \mathrm{Kg}\end{array}$ \\
\hline E4 & 7.1030 & 4.4753 & 31.788 & 0.547 & 0.059 & 0.048 & 0.007 & -0.003 & -0.002 & 2.2032 & 14.726 \\
\hline$E^{\prime} 4$ & 7.1537 & 4.4758 & 32.019 & 0.464 & 0.046 & 0.050 & 0.008 & -0.004 & ᄃo & 2. 1984 & 14.820 \\
\hline E6 & 6.8905 & 4.4749 & 30.834 & 0.498 & 0.046 & 0.065 & 0.015 & -0.002 & ᄃo & 2.1761 & 14.454 \\
\hline$E^{\prime} 6$ & 6.7822 & 4.4758 & 30.356 & 0.506 & 0.032 & 0.101 & 0.016 & -0.003 & do & 2.1381 & 14.502 \\
\hline E7 & 8.2678 & 4.4758 & 37.005 & 0.492 & 0.054 & 0.042 & 0.016 & -0.003 & do & 2.2520 & 16.698 \\
\hline$E^{\prime} 7$ & 8.2695 & 4.4752 & 37.007 & 0.602 & 0.048 & 0.033 & 0.014 & -0.002 & do & 2.2564 & 16.708 \\
\hline E9 & 9.2526 & 4.4771 & 41.425 & 0.486 & 0.052 & 0.011 & 0.005 & -0.005 & do & 2.2849 & 18.369 \\
\hline$E^{\prime} 9$ & 9.2217 & 4.4766 & 41.282 & 0.399 & 0.042 & 0.006 & 0.008 & -0.006 & do & 2.2899 & 18.223 \\
\hline
\end{tabular}

(in Carbou in ash is assumed to be elemental carbon. 


\section{Journal of Research of the National Bureau of Standards}

ments and the calibration experiments. To illustrate typical magnitudes of the individual corrections to $E_{\mathrm{s}}$, the heat capacity correction for the water filling temperature, water in the collector, and ash in the combustor for experiment $\mathrm{E} 6$ are $-0.5 \mathrm{~kJ} / \mathrm{K}$, $-1.9 \mathrm{~kJ} / \mathrm{K}$, and $+0.6 \mathrm{~kJ} / \mathrm{K}$, respectively. The definitions of the enthalpy corrections in columns 5 through 7 and in column 10 are the same as those in the corresponding columns in table 2 . The correction for incomplete combustion given in column 8 is for the presence of carbon (assumed to be elemental carbon) in the ash. The correction given in column 9 is for the formation of $\mathrm{SO}_{4}{ }^{2}(\mathrm{aq})$ rather than $\mathrm{SO}_{2}(\mathrm{~g})$.

The enthalpy of combustion per unit dry mass, $-\Delta \vec{H}_{\text {st }}$ is listed in the last column. Using pair differences, a pooled estimate $[11,12]$ of the standard deviation of a single measurement of $-\Delta \vec{H}_{\mathrm{st}}$ of "identical" processed MSW samples was calculated. The resulting $\% C V, 0.4 \%$, is the measurement error of the calorimeter with an actual sample of MSW. For comparison, the \% $\mathrm{CV}$ for measurements of $-\Delta \bar{H}_{\text {st }}$ on the corresponding pairs of "identical" gram-size samples of MSW obtained with the same bomb calorimeter used in the cellulose measurements was $0.5 \%$ [5].

Average corrections for the processed MSW measurements are listed in the fourth column of table 3. The water vaporization correction is about twice as large as that for the calibration measurement due to the longer reaction time (and consequent flow of oxygen for a longer time interval). The longer reaction time is due to the deceleration of the rate of combustion during the last stages of the reaction by the intimate contact of the noncombustible and combustible components in the highly processed MSW. This is also reflected in a much larger $\mathrm{CO}$ correction and an appreciable correction for carbon in the ash. The ash ranged from 18 to $35 \%$ of the initial sample mass.

As indicated in the third row of table 3 , an average of $81.4 \%$ of the total water formed in the combustion reaction of the processed MSW is retained in the collector. No reliable value for the hydrogen content of these samples (as in the case of cellulose) was available to compare the rotal collected water with that calculated from the empirical formula for the sample. However, the consistency of the experimental measurements can be checked by comparing the measured hydrogen contents of samples from the same day. The pooled estimate of the standard deviation of the hydrogen content of the pairs of samples was $0.045 \%$ of the dry pellet weight. Since the average hydrogen content was $5.15 \%$ of the dry pellet weight, the \% $C V$ of the measured hydrogen contents of all four sets of paired samples was $0.9 \%$.

\section{Design Basis and Performance Characteristics}

As indicated in section 2, our initial assessment of the industrial problem showed that a satisfactory solution would be obtained with a kilogram capacity calorimeter that could provide an enthalpy of combustion having an overall uncertainty of less than $1 \%$ for a single measurement. As flow calorimetry requires more auxiliary measurements than bomb calorimetry, the strategy adopted to achieve this goal was to design and operate the instrumentation so that contributions of individual errors would be held to $0.1 \%$ or less. This section documents the extent to which this strategy was achieved.

Measures are described in section 5.1 which ensure that the energy equivalent of the calorimeter for standard calorimeter conditions is the same for both calibration and "unknown" experiments. Errors associated with the corrected temperature rise are described in section 5.2, and an overall uncertainty estimate is discussed in section 5.3.

\subsection{Design Basis}

The large sample flow calorimeter described is a stirred-water, isoperibol ${ }^{6}$ calorimeter of the CoopsVan Nes design (see reference [13]). Coops and Van Nes [14] devised the "flow pattern control shield" cited in [13] which we call the flow shield, for short. The design basis for closed isoperibol calorimeters (i.e., those for which no reactants or products flow across the calorimeter boundary) has been reviewed elsewhere [13]. From a review of some previous work on calorimeter theory [15] and on the design practice and data analysis of flow calorimetry [16], we concluded that the design basis of open calorimeters (i.e., those for which reactants or products do flow across the calorimeter boundary) are essentially the same as those for closed calorimeters. In particular, to account for the enthalpy of the gases entering or leaving the calorimeter proper, the gas flow tubes should be in good thermal contact with the calorimeter water and jacket water, and the measurement junctions of the thermocouples used to monitor the temperatures of the fluids entering and leaving the

\footnotetext{
${ }^{6}$ Isoperibol indicates the presence of a constant temperature environment, i.e., a calorimeter with a constant temperature jacket.
} 


\section{Journal of Research of the National Bureau of Standards}

calorimeter should be located at the interspace. Since the oxygen entering our calorimeter is passed through coils immersed in the jacket water, the temperature of the entering gas is equal to the jacket water temperature. By virtue of the product gas heat exchange coil and the collector, the temperature of the exit gas is equal to that of the calorimeter water. From measurements with thermocouples, it was found to be within $0.1^{\circ} \mathrm{C}$ of the temperature of the calorimeter water at the location of the inner quartz oscillator thermometer.

The primary calorimeter design problem is to meet the requirement that the energy equivalent for standard calorimeter conditions be the same for both the calibration and "unknown" experiments. This design problem is referred to as the problem of equivalent sources and the associated error is referred to as the nonequivalent source error. In the following four sections (5.1.1 through 5.1.4) we review the measures taken to ensure that the nonequivalent source error is less than $0.1 \%$.

5.1.1 Flow Shield. The flow shield, F of figure 1, was introduced because it provides efficient circulation of water within the cylindrical calorimeter vessel. Its purpose [13] is to make the temperature gradients in the water of the annular space between the flow shield and the calorimeter vessel, which we shall call the liquid shell, independent of the difference in temperature gradients on the heat sources (i.e., the combustor, combustor enclosure, heat-exchange coil, and collector) between the calibration and "unknown" reactions. This requires: 1) that the calorimeter water enters the liquid shell from the stirrer port in the flow shield (the temperature of the stirred water is uniform) and, that 2) the heat that is transferred from the liquid inside the flow shield to the liquid shell by heat transfer across the flow shield be negligible in comparison with that by forced convection due to stirring. For ease of assembly and substantial reduction in cost and fabrication time, we elected to depart from the second requirement, which would require the flow shield to be, in effect, a Dewar vessel. We made the flow shield from a sheet of $1.6 \mathrm{~mm}$ thick 316 stainless steel.

To compensate for this decision, we chose to minimize the nonequivalent source error by reducing the difference in termperature gradients inside the flow shield between the calibration and "unknown" experiments as discussed in sections 5.1.2 through 5.1.4.

5.1.2 Combustor Enclosure. The calorimeter is calibrated with a pellet of solid micro-crystalline cellulose that is burned in the same location as the "unknown" sample of MSW. As a steady-state ap- proach is sufficient to analyze the equivalent source problem (see reference [13], p. 3-7), we note that, for the same rate of heat evolution by the calibration and "unknown" samples, the differences between the temperature gradients on the combustor enclosure in a calibration and "unknown" experiment are substantially less than on the combustor wall. This is due to the relatively poor heat transfer by gaseous conduction and convection between the combustor and the combustor enclosure during the main period.

5.1.3 Cellulose as a Calibrant. The only difference in the temperature gradients on the product gas heat-exchange coil is that caused by the different concentrations of water in the product gas stream from the combustion of calibration and "unknown" samples. (The differences are compared at the same rate of heat evolution.) This difference is nearly completely eliminated by using cellulose as a calibrant because paper is the major component, about 80 mass percent, of the combustible components in MSW. (The ratio of the number of moles of water to the number of moles of $\mathrm{CO}_{2}$ produced in a combustion of the MSW samples given in table 4 is 0.78 . The corresponding value for the microcrystalline cellulose samples is 0.83 .)

5.1.4 Calorimeter Water Heat Transfer. To guarantee a small equivalent source error, the flow pattern of the calorimeter water must be the same (i.e., the same heat transfer characteristics) for both the calibration and "unknown" reactions (see reference [13] p. 3-4). This requirement is met by: a) adjusting the stirring rate of stirrer, $\mathrm{H}$ of figure 1 , to be the same in every experiment with a tachometer and b) by filling the calorimeter vessel with the same volume of water at nearly the same temperature in every experiment. The point of procedure b) is that the coefficient of heat transfer between the calorimeter vessel lid and the liquid shell shall be the same in every experiment and preferably large to reduce possible errors due to differences in the coefficients of heat transfer.

The coefficient of heat transfer between the calorimeter water and the vessel lid was made large through the following three measures.

1) The "topping off" procedure described in section 4.1 was used to fill the calorimeter vessel as completely as possible, while allowing for the thermal expansion of the water during the run. Measurement of the level of the water inside the assembled calorimeter vessel established that the height of liquid in the various fillings varied by less than $1.6 \mathrm{~mm}$.

2) A $3 \mathrm{~mm}$ thick sheet of copper was bonded with epoxy cement and bolted to the underside of 
the 316 stainless steel calorimeter vessel lid to improve the radial heat transfer in the lid. The stirring action lifts calorimeter water up against the central portion of the underside of the vessel lid.

3) The stirring rate was made as large as was consistent with keeping the fluctuations in calorimeter water temperature less than $0.0001{ }^{\circ} \mathrm{C}$.

5.1.5 Nonequivalent Source Error. The nonequivalent source error was estimated from an analysis of a simple model that takes into account the temperature gradients in the calorimeter water and temperature gradients on the combustor enclosure, flow shield and calorimeter vessel. The calculated result was of the order of $\pm 0.01 \%$ of $\Delta \vec{H}_{\mathrm{st}}$. To allow for the possibility that the simplicity of the model results in an underestimate of the nonequivalent source error, we increased the estimate of the uncertainty due to this source of error to $\pm 0.05 \%$ of $\Delta \vec{H}_{\mathrm{st}}$.

\subsection{Heat Exchange Correction}

Small deviations of the measured rate of change of the calorimeter water temperature from the drift period equation, eq (2), were expected for three reasons. First, the jacket water temperature, $T_{2}(t)$, changed with time. This, in effect, causes $T_{\infty}$ to vary with time because the calorimeter is designed so that its effective environment temperature is equal to $T_{2}(t)$ to a good first approximation. Thus, $T_{\infty}$ is given by eq (4)

$$
T_{\infty}=T_{2}+p_{0} / k
$$

In eq (4), $p_{0}$ is the time rate of change of the calorimeter water due to stirring. The change in jacket temperature was allowed because the total increase of $T_{2}(t)$ from $t_{\mathrm{i}}$ to $t_{\mathrm{f}}$ is generally less than $0.2^{\circ} \mathrm{C}$ and the expected correction for this effect is of the order of $0.03 \%$ of the corrected temperature rise and should be very nearly the same for both the calibration and "unknown" experiments.

The second reason for expecting the deviations from the drift period equation is that the cooling constant, $k$, should vary with temperature because the maximum difference between the calorimeter and jacket water temperatures for our experiments is from 6 to $9{ }^{\circ} \mathrm{C}$. Nonlinear heat transfer by free convection in the interspace between the calorimeter vessel and the submarine vessel cannot be assumed to be negligible (i.e., not greater than $5 \%$ of conductive heat transfer) unless the width of the interspace is less than $1.1 \mathrm{~cm}$ (see reference [17], p. 104). The selected interspace separations, $5 \mathrm{~cm}$ on the top and $2.5 \mathrm{~cm}$ on the side of the calorimeter, were dictated by manufacturing and assembly considerations.

The third reason for expecting deviations from eq (2) is that the temperature of the calorimeter water was greater than the temperature of the jacket water throughout the last $80 \%$ of the experiment-shortly after ignition to the end of the final drift period. A slight water leak in the static O-ring seals of the various flow and thermocouple lead tubes or in the rotary O-ring seal of the drive shafts of the calorimeter stirrer or quick-cool pump would allow mass transfer by evaporation of the calorimeter water to occur which results in nonlinear heat transfer. The calorimeter was operated so that its initial temperature was within $0.3^{\circ} \mathrm{C}$ of the jacket in order to reduce the time required to reach a steady initial drift rate and, thus, keep the time duration of an experiment within tolerable limits.

A study of the deviations from eq (2), which is often called a Newton's cooling law test, was made in the usual manner [18]. The calorimeter was forced to pass through a sequence of drift periods having average calorimeter water temperatures spanning the range of operation. This was accomplished by assembling the calorimeter vessel in the usual manner except that a cooling coil was placed inside the bottom port of the flow shield and the calorimeter water was preheated to $10^{\circ} \mathrm{C}$ warmer than the jacket water. The supply and exit lines of the cooling coil leave and enter the calorimeter through auxiliary chimneys (normally capped) in the calorimeter vessel lid. The lines end in quickdisconnect couplers located just outside the chimneys. After the first equilibration period of slightly more than two hours (only the last 30 minutes is the actual drift period), the supply and exit couplers of the cooling coil were connected to a chilled water supply, the calorimeter water was cooled about $2{ }^{\circ} \mathrm{C}$, the supply and exit couplers were disconnected, and another equilibrium period was started. The quick-cool pump was run throughout the entire test. No oxygen was supplied to the calorimeter in the test.

Analysis of the results of the above Newton's cooling law test shows that the data can be represented by eqs (5a) through (5d).

$$
d T_{1}(t) / d t=p_{0}-k_{0}(1+f(\beta)) \beta
$$

where

$$
\begin{aligned}
\beta & =T_{1}(t)-T_{2}(t) \\
f(\beta) & =a_{0} \beta+\mathrm{a}_{1} \beta^{2} ; 10^{\circ} \mathrm{C} \geqslant \beta \geqslant 1.5^{\circ} \mathrm{C}
\end{aligned}
$$




\section{Journal of Research of the National Bureau of Standards}

$$
f(\beta)=0 \quad ; 1.5^{\circ} \mathrm{C} \geqslant \beta \geqslant-3^{\circ} \mathrm{C}
$$

In eqs (5), $p_{0}, k_{0}, a_{0}$ and $a_{1}$ are positive constants. The parameter $T_{2}(t)$ is the jacket water temperature. The eqs (5) are identical with eq (2) if $\mathrm{T}_{\infty}$ is given by eq (4) and the cooling constant, $k$, is replaced with the temperature dependent expression:

$$
k=k_{0}(1+f(\beta)) .
$$

Equation (5a) indicates that heat transfer between the calorimeter and the jacket is nonlinear and eqs $(5 \mathrm{c})$ and $(5 \mathrm{~d})$ indicate that the larger portion of this nonlinearity is probably due to heat transfer by evaporation of the calorimeter water and condensation on the inner surface of the submarine vessel (i.e., when $\beta>0$ ) rather than heat transfer by free convection. If free convection predominated, the observed value of $f(\beta)$ would tend to be symmetric around $\beta=0$. The estimated contribution of heat transfer by free convection to $f(\beta)$ is less than $25 \%$ of the $f(\beta)$ used to estimate the maximum correction to $\Delta T_{\mathrm{c}}$ (see below). The estimated contribution of radiative heat transfer to the same $f(\beta)$ is about $2 \%$. The nonlinear heat transfer is taken into account by a temperature dependent cooling constant as shown by eq (6). The variation of the jacket water temperature with time is taken into account by the second $\beta$ on the right hand side of eq (5a) independent of whether $f(\beta)$ is or is not zero.

The correction, $\delta \Delta T_{c}$, to be added to the corrected temperature rise calculated according to eq (3) to yield the corrected temperature rise when eqs (4) and (6) are inserted into eq (3) was carried out by calculating $p_{0}$ and $k_{0}$ of eqs (5) from. $k$ and $T_{\infty}$ derived from a fit of the drift data using eq (2). he parameter $f(\beta)$ was approximated by setting $a_{1}=0$ and increasing $a_{0}$ equal to 0.067 because this provides an upper bound to $f(\beta)$ for all the data obtained in the Newton's cooling law test and simplifies the calculation of the correction. Refitting the drift period data with eqs (5) is not necessary because the value of $k$, about $3 \times 10^{-4} \mathrm{~min}^{-1}$, is sufficiently small that the fit of the integral form of eq (2) to the experimental drift data amounts to a straight line fit of the temperature as a function of time in each drift period. The expression for $\delta \Delta T_{\mathrm{c}}$ can be derived by a straightforward extension of the arguments used to derive eqs e7-e11 of reference [13].

A summary of the results of the corrections to the corrected temperature rise for six experiments is given in table 5 for two calibration experiments and for four "unknown" experiments (two for each type of MSW sample, unprocessed and processed). The second through fourth columns of table 5 list the corrected temperature rise of the calorimeter, the observed temperture rise of the calorimeter, and the corresponding observed change in temperature of the jacket water between $t_{\mathrm{i}}$ and $t_{\mathrm{f}}$, respectively. The fifth and sixth columns list the contribution to $\delta \Delta T_{\mathrm{c}}$ due to the change in jacket water temperature, and to the variation of the cooling constant with temperature. The next to last column is the total $\delta \Delta T_{\mathrm{c}}$ and the last column is $\delta \Delta T_{\mathrm{c}}$ expressed as a percent of $\Delta T_{\mathrm{c}}$. It can be seen that the total correction to $\Delta T_{\mathrm{c}}$ is $+0.04 \%$ or less and the net correction for "unknown" experiments is expected on the average to be less than $0.02 \%$. Thus, the net correction is small in comparison to $0.1 \%$.

Apart from the change in jacket water temperature with time and the change in cooling constant with $\beta$, the drift period data of the experiments are remarkably normal. For example, during the drift periods, the thermocouples indicate that compo-

\begin{tabular}{|c|c|c|c|c|c|c|c|}
\hline \multirow[t]{2}{*}{ Experiment } & \multirow[t]{2}{*}{$\Delta T_{\mathrm{c}}^{(a)}$} & \multirow[t]{2}{*}{$\Delta T_{1}^{(b)}$} & \multirow[t]{2}{*}{$\Delta T_{2}^{(\mathrm{c})}$} & \multicolumn{3}{|c|}{$\delta \Delta T_{\mathrm{c}}^{(\mathrm{d})}$} & \multirow[t]{2}{*}{$\left(\delta \Delta T_{\mathrm{c}} / \Delta T_{c}\right)$} \\
\hline & & & & Jacket Water ${ }^{(e)}$ & Cooling Constant ${ }^{(n)}$ & Total & \\
\hline \multicolumn{8}{|l|}{ Sample } \\
\hline No. & ${ }^{\circ} \mathrm{C}$ & ${ }^{\circ} \mathrm{C}$ & ${ }^{\circ} \mathrm{C}$ & ${ }^{\circ} \mathrm{C}$ & ${ }^{\circ} \mathrm{C}$ & ${ }^{\circ} \mathrm{C}$ & $\%$ \\
\hline Cellulose (35) & 8.9142 & 8.5746 & 0.1872 & 0.0050 & +0.0081 & +0.0031 & +0.035 \\
\hline Cellulose (36) & 9.4261 & 9.0560 & 0.1832 & -0.0049 & +0.0086 & +0.0037 & +0.039 \\
\hline Processed MSW (E'9) & 9.2217 & 8.8160 & 0.2379 & -0.0065 & +0.0064 & -0.0001 & -0.001 \\
\hline Processed MSW (E6) & 6.8905 & 6.6412 & 0.1556 & -0.0039 & +0.0058 & +0.0019 & +0.028 \\
\hline Unprocessed MSW (A4) & 9.6576 & 9.2598 & 0.1994 & -0.0059 & +0.0084 & +0.0025 & +0.026 \\
\hline Unprocessed MSW (B2) & 7.1685 & 6.9262 & 0.1235 & -0.0032 & +0.0060 & +0.0028 & +0.040 \\
\hline \multicolumn{8}{|c|}{ (a) Corrected temperature rise according to eq (3). } \\
\hline \multicolumn{8}{|c|}{ (b) Observed temperature rise, calorimeter water. } \\
\hline \multicolumn{8}{|c|}{ (c) Observed change in temperature of the jacket water. } \\
\hline \multirow{2}{*}{\multicolumn{8}{|c|}{ (d) Correction to be added to $\Delta T_{\mathrm{c}}$ for cliange in jacket water temperature and temperature dependence of the cooling constant. }} \\
\hline & lange in $\mathrm{j}$ & water ten & & & & & \\
\hline
\end{tabular}

Table 5. Correction for change in jacket temperature and cooling constant. 
nents of the combustor and the various parts of the calorimeter and liquid shell are isothermal to between 0.1 and $0.03^{\circ} \mathrm{C}$. This is true even when $\beta$ is about $9.5{ }^{\circ} \mathrm{C}$ and in spite of the enormous size of the calorimeter. (Even during the main period, the maximum temperature difference in the liquid shell near the top and bottom of the water flow shield is only $0.25^{\circ} \mathrm{C}$.) The exponential decay of the calorimeter temperature in the approach to the final drift period is normal in the sense that the decay time constant, 15 minutes, is a small multiple of the water circulation time of 5.4 minutes.

\subsection{Uncertainty Estimates}

Estimates of the systematic errors in the experiments are given in table 6 . The first column contains the symbol in eq (1) associated with the source of the error. The second column lists the type of uncertainty. The values given in the third column are the net effect of the estimated systematic error on $-\Delta \vec{H}_{\text {st }}$ for dry MSW samples. Unless stated otherwise, the errors are the same for processed and unprocessed MSW samples.

The error estimate given in the first row is the overall error cited in section 5.1.5. The error given in the second row is the average calculated from table 5 for processed MSW. The corresponding value for unprocessed MSW is $0.00 \%$.

The value of the error given in the third row is the uncertainty associated with the assumption that the residual moisture in the kilogram-size flow calorimeter samples of processed MSW and cellulose is the same as in the gram-size test samples. Moisture content determinations were only made on the gram-size test samples which were taken from each parent and stored in moisture proof containers. The moisture was determined by ASTM method E790-81, which involves weighing the test samples and then drying the samples in loose form at $105{ }^{\circ} \mathrm{C}$ for an hour in a mechanical convection oven. Samples were weighed after they cooled in a desiccator. The moisture content of each parent sample was corrected for any weight change between the time of its riffling and the time the parent was pelleted and combusted.

For unprocessed MSW samples, the error in the third row is the uncertainty associated with the assumption that the moisture is equivalent to that which would have been obtained had the sample been processed and its moisture content determined with a gram-size test sample. The moisture content of each increment of unprocessed MSW was determined by drying the entire increment in loose form in a large open pan for 12 hours at
Table 6. Estimates of systematic error.

\begin{tabular}{|c|c|c|}
\hline Quantity & $\begin{array}{l}\text { Source of Error } \\
\text { Type of Uncertainty }\end{array}$ & $\begin{array}{c}\text { Percent Effect } \\
\quad \text { on } \\
-\vec{H}_{\mathrm{si}} \text { of } \mathrm{MSW} \\
\end{array}$ \\
\hline 1. $E$ & Nonequivalent sources & \pm 0.05 \\
\hline 2. $\Delta T_{c}$ & $\begin{array}{l}\text { Change in jacket water temperature } \\
\text { and } \\
\text { Temperature dependence of the } \\
\text { cooling constant (see } \delta \Delta T_{c} \text { ). }\end{array}$ & $-0.02^{(4)\}}$ \\
\hline 3. $M$ & $\begin{array}{l}\text { Moisture same in processed MSW } \\
\text { parent and gram-size test samples } \\
\text { or } \\
\text { Moisture same in unprocessed MSW } \\
\text { and gram-size test samples. }\end{array}$ & \pm 0.20 \\
\hline 4. $\Delta H_{\mathrm{v}}$ & Oxygen flow rate. & \pm 0.03 \\
\hline 5. $\Delta H_{\mathrm{g}}$ & Oxygen flow rate. & \pm 0.00 \\
\hline 6. $\Delta H_{\mathrm{ic}}$ & $\begin{array}{l}\text { Oxygen flow rate, effect on mea- } \\
\text { surement of } \mathrm{CO}(\mathrm{g}) \text {. }\end{array}$ & \pm 0.01 \\
\hline 7. $\Delta H_{\vee}$ & $\begin{array}{l}\text { Input and output gas moler flow } \\
\text { rates equal. }\end{array}$ & 0.002 \\
\hline 8. $\Delta H_{\mathrm{ic}}$ & $\begin{array}{l}\text { Input and output gas molar flow } \\
\text { rates equal, effect on measurements } \\
\text { of } C O(g)\end{array}$ & -0.001 \\
\hline 9. $\Delta H_{\text {ic }}$ & $\begin{array}{l}\text { Carbon in ash is cellulose rather } \\
\text { than free carbon. }\end{array}$ & $0.01^{(b)}$ \\
\hline 10. $M$ & $\begin{array}{l}\text { Dispersion of sample in combustion } \\
\text { zone. }\end{array}$ & \pm 0.05 \\
\hline 11. $\Delta H_{\mathrm{x}}$ & $\begin{array}{l}\text { Kinetic energy loss and } \\
\text { Joule-Thompson cooling. }\end{array}$ & -0.000 \\
\hline 12. & $\begin{array}{l}\text { Heat of wetting: } \\
\text { Effect on gram minus kilogram } \\
\text { samples of processed MSW } \\
\text { Effect on unprocessed minus } \\
\text { processed kilogram samples }\end{array}$ & $\begin{array}{l}-0.000^{(\mathrm{c})} \\
-0.002^{(\mathrm{s})}\end{array}$ \\
\hline
\end{tabular}

Total Systematic Error

$=0.37$

(a) applies to processed MSW, value for unprocessed is $0.00 \%$.

(b) applies to processed MSW, value for unprocessed is $0.02 \%$.

(c) error in appropriate average difference in calorific value, expressed as \% of average processed calorific value.

$105^{\circ} \mathrm{C}$ in a large, mechanical convection oven. The sample was weighed after it had cooled (about one hour later). Then, a known mass of water was added to the sample to return the moisture content to about 3 to $4 \%$, the sample was pelleted, weighed, and burned. The water added after drying improves the cohesion of the pellet. The error estimate is believed to take into account moisture pick-up during the cooling of the increment (stud- 
ied in an auxiliary experiment) as well as other possible errors due to differences between this procedure and that employed for the gram-size test samples.

It should be noted that the "moisture" error in the third row does not involve questions about absolute moisture content. Only errors associated with differences in moisture content are pertinent to the results (see end of section 2). Further, vaporization of different amounts of moisture initially present in a sample during different combustion runs contributes no error since the dew point of the gases leaving the calorimeter is continuously monitored.

The error values given in the fourth through sixth rows are due to the estimated uncertainty in the total oxygen flow rate which is estimated to be, at most, $5 \%$. This estimate is believed to take into account that the total flow rate is the sum of the measured flow rates through six flow meters as well as assumptions about the nonlinearity of some of the uncalibrated flow meters.

The error values given in the seventh and eighth rows are associated with the error in the assumption that the total input oxygen and output product gas flow rates are equal. The correct product gas flow rate was calculated from the sum of the input oxygen flow rate, the flow rate of water vapor in the product gas (based on the measured water concentration), and the correction for the moles of oxygen consumed in the production of water. The latter correction was estimated from the measured $\mathrm{CO}_{2}$ concentration in the product gas and the stoichiometric coefficients of the standard combustion reaction using an empirical formula for MSW. The error is very small because the combustible fraction of the MSW is such that the amount of oxygen consumed to oxidize hydrogen to water is small and most of the water produced in the reaction remains trapped in the collector.

The error value given in the ninth row is the effect of treating the carbon component of the ash as cellulose rather than elemental carbon. In the 10th row, we list the error associated with the amount of sample (about $1 \mathrm{~g}$ ) that may be dispersed by the flowing gas prior to ignition and does not get burned after the reaction starts.

The error in the 11 th row is that associated with the assumption that $\Delta H_{\mathrm{x}}$ of eq (1) is zero. The magnitude of the contribution to $\Delta H_{\mathrm{x}}$ by the expansion cooling (i.e., Joule-Thompson cooling) of the inlet oxygen was estimated from the calculated mean pressure drop of the inlet oxygen $(0.34 \mathrm{~atm})$ to be $34 \mathrm{~J} / \mathrm{min} \times \Delta t$ where $\Delta t$ is the time during which the inlet oxygen flow rate is $270 \mathrm{~L} / \mathrm{min}$. The kinetic energy loss contribution to $\Delta H_{\mathrm{x}}$ is estimated to be $-31 \mathrm{~J} / \mathrm{min} \times \Delta t$. The net effect on $-\Delta \bar{H}_{\mathrm{st}}$ is $0.000 \%$.

The error in the 12th row is that associated with the heat of wetting of the samples. The mean difference in the moisture content of the gram-size test samples and the same parent processed MSW samples is $0.00 \%$. The mean difference in the moisture contents of the unprocessed MSW minus that of the corresponding "identical" processed increments is $-0.07 \%$. Using the data analysis given in reference [19] and assuming the combustible components are given cellulose the $-0.07 \%$ moisture difference, for example, corresponds to an estimated systematic error of $-0.002 \%$ to be added to the average difference of $-0.5 \%$ (see section 2).

The overall systematic error given at the bottom of the table is calculated by adding the individual errors algebraically. No error is given for degradation association with preparation (milling) of the processed MSW samples from the unprocessed MSW samples since the purpose of one of the sets of the experiments was to determine this change. The results as cited in section 2 indicate the error is of the order $-0.5 \%$ of $-\Delta \vec{H}_{\mathrm{st}}$.

The standard deviation of a single calibration experiment is $0.2 \%$ of the average energy equivalent. It is worth noting that this \% CV is not primarily due to the variation in the amount of water added to the calorimeter. Separate tests of the filling technique were carried out using a load cell to intercompare the mass of the calorimeter vessel filled with water with a $6000 \mathrm{~kg}$ tare mass. The $\% \mathrm{CV}$ of the ratio of the mass of the tare divided by the mass of the full (water) calorimeter vessel was $0.01 \%$.

The standard deviation of a single measurement of $\Delta \bar{H}_{\mathrm{st}}$ for MSW samples is $0.4 \%$ of $\Delta \bar{H}_{\mathrm{st}}$. Thus, the overall uncertainty in a single measurement of $\Delta \bar{H}_{\mathrm{st}}$ is $\pm 0.8 \%$, which is the sum of the standard deviation of $\pm 0.4 \%$ and the systematic error of $\pm 0.37 \%$.

\section{Conclusions}

1) A calorimeter capable of determining the enthalpy of combustion of kilogram-size samples of MSW has been successfully constructed and operated. The number of experiments carried out to date (April 1986) is approximately 60.

2) Samples have been successfully burned in flowing oxygen near atmospheric pressure. Combustion of the organic portion of the $\mathrm{MSW}$ to $\mathrm{CO}_{2}$ and $\mathrm{H}_{2} \mathrm{O}$ is complete, in terms of the enthalpy of 
combustion, to $99.9+\%$ even when the initial mass contains 35 mass \% of noncombustible components in intimate contact with the combustible components. In the absence of these noncombustible components, oxidation is complete to $99.99 \%$.

3) The above degree of completeness of oxidation is accomplished in spite of the fact that the sample is burned as a single, multi-kilogram pellet rather than in loose form. Combustion of the material in pellet form has the major advantage of simplifying the quantitative collection of ash and also restricts the reaction zone to a smaller volume.

4) The $\% C V$ for calibration experiments is $0.2 \%$ and for measurements of $-\Delta \vec{H}_{\mathrm{st}}$ of "unknown" MSW samples is $0.4 \%$.

5) Because of cost and time considerations, we departed from a number of design and operation dictums for stirred-water calorimeters with a constant-temperature jacket. Because of manufacturing considerations, the calorimeter design did not exclude heat transfer by free convection with the surrounding jacket. The calorimeter was operated at a temperature above its jacket temperature to reduce the duration of an experiment. The jacket water temperature was allowed to drift about $0.2^{\circ} \mathrm{C}$. An error analysis shows that the effect of all these departures is each less the $0.1 \%$.

6) The overall uncertainty of a single calorimetric result is judged to have an absolute value of less than $1 \%$ by virtue of the calorimeter having been designed and operated so that the cumulative contribution of the individual possible sources of systematic error is of the order of $\pm 0.4 \%$.

Technical support for this project was provided by members of the ASME Research Committee on Industrial and Municipal Wastes, the ASME Performance Test Code Committee (PTC-33) on Large Incinerators, and the ASTM Committee (E-38) on Resource Recovery and its Subcommittee E-38.01 on Energy. These committees helped to identify this work as an important industrial research need.

We acknowledge the contributions of the following members of the Chemical Thermodynamics Division of the Center for Chemical Physics: J. C. Colbert performed the bomb calorimetry measurements, T. J. Buckley assisted in the data reduction computations, and S. S. Bruce assisted in the execution of the experiments. R. C. Paule of the NBS National Measurement Laboratory provided statistical consultation.

\section{References and Notes}

[1] Kirklin, D. R.; J. C. Colbert, P. H. Decker, A. E. Ledford, Jr., R. V. Ryan, and E. S. Domalski, The variability of municipal solid waste and its relationship to the determination of the calorific value of refuse-derived fuels, Resources and Conservation 9, 281 (1982); see also Part A of NBSIR 82-2491, U.S. Natl. Bur. Stand,, Washington, DC (1982).

[2] Reilly, M. L.; K. L. Churney, D. R. Kirklin, A. E. Ledford, and E. S. Domalski, An oxygen flow calorimeter for kilogram-size samples of municipal solid waste, Part I. A 25 gram capacity combustion flow calorimeter for determining the calorific value of refuse-derived fuels, Resources and Conservation 8, 147 (1982).

[3] Domalski, E. S.; K. L. Churney, M. L. Reilly, D. R. Kirklin, A. E. Ledford, and D. D. Thorton, 25 gram capacity combustion flow calorimeter, NBSIR 84-2457, U.S. Natl. Bur. Stand., Washington, DC (1982).

[4] Ledford, A. E.; R. V. Ryan, M. L. Reilly, E. S. Domalski, and $\mathrm{K}$. L. Churney, An oxygen flow calorimeter for kilogram-size samples of municipal solid waste. Part II. Trial combustions of kilogram-size samples of municipal solid waste, Resources and Conservation 8, 159 (1982).

[5] Churney, K. L.; E. S. Domalski, A. E. Ledford, J. C. Colbet, S. S. Bruce, T. J. Buckley, R. C. Paule, and M. L. Reilly, Assessing the credibility of the calorific value of municipal solid waste, NBSIR 84-2825 (DoE), U.S. Natl. Bur. Stand., Washington, DC (1984).

[6] Prosen, E. J.; F. W. Maron and F. D. Rossini, Heat of isomerization of the two butadienes, J. Res. Natl. Bur. Stand. 42, 269 (1949).

[7] Sunner, S., Basic principles in combustion calorimetry, Chap. 2 in Combustion Calorimetry, Experimental Chemical Thermodynamics, Volume 1, S. Sunner and M. Manson, editors, Pergamon Press: Oxford (1979).

[8] Goldberg, R. N.; R. L. Nuttall, E. J. Prosen, and A. P. Brunetti, Digital data acquisition and computer computation applied to calorimetric experiments, Natl. Bur. Stand. (U.S.) Report 10-437, June 1971. See Program TEMPQTZ. See also Steckler, D. K.; R. N. Goldberg, R. B. Tewari, and T. J. Buckley, Computer software for the acquisition and treatment of calorimetric data, NBS Technical Note 1224, U.S. Natl. Bur. Stand., Washington, DC (1986).

[9] Vanderzee, C. E., Reduction of results to the isothermal calorimetric process and to the standard-state process in solution-reaction calorimetry, J. Chem. Thermodynamics 13, 947 (1981); Evaluation of corrections from temperature-time curves in isoperibol calorimetry under normal and adverse operating conditions, ibid. 13, 1139 (1981).

[10] Equations (9a) and (9b) and the enthalpy change of reaction (6) of reference [3] are in error. The correct expressions are:

$$
\begin{aligned}
& \left.Z \text { (soln, } T_{\mathrm{i}}\right)=\left(N_{7}+N_{3}^{\mathrm{i}}-N^{[}\right) \mathrm{H}_{2} \mathrm{O}\left(g, T_{1}\right)+ \\
& {\left[Z-\left(N_{7}+N !-N_{3}^{i}\right) \mathrm{H}_{2} \mathrm{O}\right](\text { soln, } T) ;} \\
& p=1 \mathrm{~atm} . \\
& Z=N_{9} \mathrm{HCl}+N_{10} \mathrm{H}_{2} \mathrm{O} . \\
& \Delta H \text { for eq (6) when } N_{4}=1 \text { is }-282.98 \mathrm{~kJ} / \mathrm{mol} .
\end{aligned}
$$

It should be noted that this work, $\Delta H_{\mathrm{g}}$ was calculated as the time integral of the expression $\dot{N}_{\text {in }} C_{2}\left(T_{1}-T_{2}\right)$ where $\dot{N}_{\mathrm{t}}$ is $N_{\mathrm{t}}+N_{2}$ of reference [3], $\dot{N}_{\mathrm{tr}}$ is the total molar input 
oxygen flow rate, $C_{2}$ is the heat capacity of oxygen, and $T_{2}$ is the jacket water temperature. This differs by less thn $2 \%$ from $\Delta H_{g}$ given by eqs (8) of reference [3], as determined from the results of the calculations for entry 7 of table 6. It should also be noted that in the present work both $N_{10}-N_{7}$ as well as $N_{7}$ are measured in each experiment and no gas flow is permitted during the final drift period (see p. 23, reference [3]).

[11] Mandel, J., and R. C. Paule, Interlaboratory evaluation of a material with unequal numbers of replicates, Analytical Chemistry 42, 1194 (1970) and Correction, Analytical Chemistry 43, 1287 (1971).

[12] Mandel, J., and R. C. Paule, Statistical analysis of sampling and measurement errors in the characterization of refuse-derived fuel, Proceedings of the Second International Conference on Materials and Energy from Refuse, Antwerp, Belgium, p. 6.25 (1981).

[13] Mosselman, C., and K. L. Churney, Calibration of combustion calorimeters, Chap. 3 in Combustion Calorimetry, Experimental Chemical Thermodynamics, Volume 1, S. Sunner and $M$. Mansson, editors, Pergamon Press: Oxford (1979).

[14] Coops, J., and K. Van Nes, Researches on heat of combustion II. Internal lag and method of stirring in isothermally jacketed calorimeters, Rec. Trav. Chim. 66, 131 (1947).

[15] Churney, K. L.; E. D. West, and G. T. Armstrong, A cell model for isoperibol calorimeters, NBSIR 73-184, U.S. Natl. Bur. Stand. (1973).

[16] Pilcher, G. Oxygen flame calorimetry, Chap. 14 in Combustion Calorimetry, Experimental Chemical Thermodynamics, Volume 1, S. Sunner and M. Mansson, editors, Pergamon Press: Oxford (1979).

[17] Ginnings, D. C., and E. D. West, Principles of calorimetric design, Chap. 4 in Experimental Thermodynamics, Volume 1, Calorimetry of Non-Reacting Systems, J. P. McCullough and D. W. Scott, editors, Plenum Press: New York (1968).

[18] Churney, K. L., and G. T. Armstrong, Studies in bomb calorimetry. A new determination of the energy of combustion of benzoic acid in terms of electrical units. J. Res. Natl. Bur. Stand. 72A, 453 (1968).

[19] Colbert, J. C.; He Xiheng and D. R. Kirklin, Enthalpy of combustion of microcrystalline cellulose, J. Res. Natl. Bur. Stand. 86, 665 (1981). 